\title{
Reflexive attraction in comprehension is selective
}

Dan Parker ${ }^{1}$ and Colin Phillips ${ }^{2,3}$

(C) 2017. This manuscript version is made available under the Elsevier user license http://www.elsevier.com/open-access/userlicense/1.0/ 


\begin{abstract}
Many studies have shown that attraction effects are consistently found for linguistic dependencies like subject-verb agreement, e.g., * The key to the cabinets are on the table. However, not all dependencies are equally susceptible to attraction. A parade case involves reflexive-antecedent dependencies, which rarely show attraction effects. The contrast between agreement and reflexives with respect to attraction has motivated various proposals regarding the memory architecture for the parser, including the use of qualitatively different access mechanisms or the selective use of morphological features as retrieval cues for different dependencies. In this paper, we show how to systematically induce attraction effects for reflexives in three eye-tracking experiments. Furthermore, we show based on computational simulations that it is possible to derive both the presence and absence of reflexive attraction from the same retrieval mechanism, based on the ACT-R architecture. We then propose an account of why agreement and reflexives are differentially susceptible to attraction, based on the predictability of the dependency.
\end{abstract}




\section{Introduction}

The task of building a sentence structure requires mechanisms for encoding a structured representation in memory and accessing specific elements of that representation to guide further elaboration. The effects of grammatically irrelevant items on real-time linguistic dependency formation have proven to be a useful tool for understanding these mechanisms. For instance, to determine whether the reflexive anaphor themselves in (1) is appropriately licensed, memory access mechanisms must recover the encoding of the subject noun executive, while avoiding interference from grammatically irrelevant items that match the agreement features of the reflexive, such as the embedded plural noun managers (example (1) is from Dillon, Mishler, Sloggett, \& Phillips, 2013).

(1) *The executive who oversaw the managers doubted themselves on most decisions.

Previous research has shown that grammatically irrelevant items rarely interfere during reflexive licensing (e.g., Clifton, Frazier, \& Deevy, 1999; Cunnings \& Sturt, 2014; Dillon et al., 2013; Nicol \& Swinney, 1989; Sturt, 2003; Xiang Dillon, \& Phillips, 2009; but cf. Cunnings \& Felser, 2013; Patil, Vasishth, \& Lewis, 2016). However, retrieval processes do not always avoid grammatically irrelevant items during dependency formation. For instance, Dillon and colleagues found that retrieval for subject-verb agreement licensing in closely matched sentences like (2) is susceptible to interference ('attraction') from the grammatically irrelevant plural noun managers, giving rise to an 'illusion of acceptability' (Dillon et al., 2013; see also Clifton et al., 1999; Patson \& Husband, 2015; Pearlmutter, Garnsey, \& Bock, 1999, Phillips, Wagers, \& Lau, 2011; Tanner, Nicol, \& Brehm, 2014; Tucker, Idrissi, \& Almedia, 2015; Wagers, Lau, \& Phillips, 2009).

(2) *The executive who oversaw the managers were dishonest.

The contrast between agreement and reflexive licensing with respect to attraction is striking, since subject-verb agreement and reflective-antecedent dependencies have superficially identical agreement constraints and retrieval in both cases targets the same structural position, i.e., the 
subject of the current clause. The reasons for this contrast remain unresolved, and we attempt to address this puzzle in the current study.

\section{Interference effects in comprehension}

Research on memory access in sentence comprehension has identified two distinct types of interference. The first type is referred to as 'inhibitory interference', and manifests as increased processing difficulty during dependency formation. For example, Van Dyke and Lewis (2003) tested sentences like those in (3) and observed processing difficulty reflected in longer reading times at the verb complaining in (3b) relative to (3a). Van Dyke and Lewis interpreted this effect as a case of inhibitory interference, where the overlap in animacy between the two subject noun phrases (NPs) the resident and the neighbor made it relatively difficult for comprehenders to retrieve the target subject the resident during subject-verb binding in (3b). Similar effects have been observed across a number of other studies (e.g., Fedorenko, Babyonyshev, \& Gibson, 2004; Gordon, Hendrick, \& Johnson, 2001, 2004, 2006; Van Dyke, 2006; Van Dyke, 2007).

(3) a. The worker was surprised that the resident who was living near the dangerous warehouse was complaining about the investigation.

b. The worker was surprised that the resident who was living near the dangerous neighbor was complaining about the investigation.

The second type of interference, known as 'attraction' (also called 'intrusion' or 'facilitatory interference'), manifests as eased processing and increased acceptability during dependency formation, relative to sentences that should be equally acceptable or unacceptable. Comprehenders frequently experience attraction during subject-verb agreement licensing. For instance, Wagers and colleagues used self-paced reading and speeded-acceptability judgments to examine the processing of grammatical and ungrammatical subject-verb agreement dependencies like those in (4).

(4) a. The key to the cabinet(s) unsurprisingly was rusty ...

b. *The key to the cabinet(s) unsurprisingly were rusty ... 
Wagers and colleagues found that in grammatical sentences like (4a), the number marking on the plural distractor cabinets did not impact acceptability judgments or reading times after the verb. However, in ungrammatical sentences like (4b), the presence of the plural distractor cabinets, which matched the number of the verb were, increased rates of acceptance and facilitated reading times after the verb, relative to an ungrammatical condition with the singular cabinet.

Wagers and colleagues argued that the facilitation observed in sentences like (4b) was due to incorrect retrieval of the plural distractor, e.g., cabinets, which matches the plural retrieval cue at the verb. Wagers and colleagues offered two suggestions for how a retrieval-based account could capture the grammatical asymmetry observed in (4). One possibility is that retrieval functions as an error-driven repair mechanism that is triggered by the detection of an agreement violation. In (4), the subject NP predicts the number of the verb. When the verb form violates this prediction, as in (4b), the parser engages a cue-based retrieval at the verb to recover a number matching noun to license agreement. The distractor cabinets should sometimes be incorrectly retrieved because it matches the verb in number, leading to the false impression that agreement is licensed. In the grammatical condition (4a), the verb fulfills the number prediction made by the subject $\mathrm{NP}$, and therefore retrieval is not engaged, reducing the possibility for attraction. Another possibility is that retrieval is always engaged, regardless of grammaticality. On this view, no attraction is expected in the grammatical condition, since the presence of a fully matching target NP should strongly outcompete partial matches. These accounts contrast with earlier proposals that attraction results from the "percolation" of plural features from the distractor to the subject noun (Eberhard, Cutting, \& Bock, 2005; Franck, Vigliocco, \& Nicol, 2002; Pearlmutter et al., 1999), which incorrectly predict grammatically symmetrical attraction effects.

Attraction effects are not limited to subject-verb agreement and have also been reported for negative polarity item (NPI) licensing and null subject licensing (e.g., Drenhaus, Saddy, \& Frisch, 2005; Parker \& Phillips, 2016; Parker, Lago, \& Phillips, 2015; Vasishth, Brüssow, \& Lewis, 2008; Xiang et al., 2009). Across most of these studies, the facilitation effects were interpreted as the behavioral signature of faulty memory retrieval mechanisms. ${ }^{1}$

\footnotetext{
${ }^{1}$ Xiang et al. (2009) argued that the facilitation effect observed for NPI licensing does not reflect misretrieval, but rather over-application of the pragmatic licensing mechanisms that are responsible for normal NPI licensing.
} 
Inhibitory interference and attraction have different behavioral signatures and license different conclusions about memory access in sentence comprehension. Inhibitory interference occurs in multiple match contexts, where the target and distractor both match some of the retrieval cues. Inhibitory interference could reflect a number of different underlying mechanisms, such as feature-overwriting during encoding (Nairne, 1998, 1990) or the use of degraded cues in retrieval (Anderson, 1974). By contrast, attraction arises when the target and distractor are distinct in feature content, but neither is a perfect match to the retrieval cues. In the case of attraction, retrieval of a partially matched distractor can ease processing of an unlicensed dependency by preventing a total retrieval failure. Unlike inhibitory interference, attraction has been interpreted as clear evidence for the retrieval of grammatically irrelevant items during dependency formation (see Dillon 2011, for discussion).

\section{Contrasting attraction profiles}

Attraction effects are consistently found for subject-verb agreement, but not for reflexive licensing. The contrast between agreement and reflexives with respect to attraction is evident in a recent study by Dillon et al. (2013). Dillon and colleagues used eye-tracking while reading to compare subject-verb agreement and reflexive licensing in closely matched sentences like (5), where the auxiliary verb in (5a) and the reflexive in $(5 b)$ must agree in number with the same subject, i.e., the new executive.

(5) a. The new executive who oversaw the middle manager(s) apparently was | were dishonest about the company's profits.

b. The new executive who oversaw the middle manager(s) apparently doubted himself | *themselves on most major decisions.

Dillon and colleagues found that the processing of the ungrammatical plural verb were in (5a) was susceptible to attraction from the plural distractor managers, but that the processing of the ungrammatical plural reflexive themselves in (5b) was not. In the reflexive conditions, comprehenders were sensitive only to the number (mis)match between the reflexive and the target subject NP the new executive. 
The lack of attraction effects for reflexives is consistent across experimental measures and structural configurations. It has been replicated using eye-tracking while reading (Cunnings \& Sturt, 2014) and event-related potentials (ERPs) (Xiang et al., 2009). In an early study on reflexive processing, Sturt (2003) found no attraction effects in contexts that involved a manipulation of gender agreement. In two eye-tracking experiments, Sturt manipulated the (stereotypical) gender match between two potential licensors and the reflexive in configurations where the distractor appeared before the target subject (Experiment 1) or after (Experiment 2). Across both configurations and most measures, comprehenders showed sensitivity only to the gender (mis)match of the target subject. ${ }^{2}$ A small number of studies have reported interference in reflexive processing (e.g., Cunnings \& Felser, 2013; King, Andrews, \& Wagers, 2012; Patil et al., 2016). But the effects reported in these studies are smaller and less consistent than the effects reported for agreement, and some reflect inhibitory interference effects, or involve reflexives in non-canonical positions, which may be subject to different licensing conditions than those that govern direct object reflexives.

The contrasting retrieval profiles for agreement and reflexives have important consequences for theories of cue-based memory retrieval. A major question for cue-based parsing models concerns how grammatical constraints relate to retrieval cues. In English, agreement and reflexive-antecedent dependencies both require morphological feature concord between elements in the dependency, so the use of morphological features as retrieval cues is motivated. If there is a transparent mapping from grammatical constraints to retrieval cues, then agreement and reflexives should deploy morphological features as retrieval cues in the same fashion. However, the contrasting retrieval profiles for reflexives and agreement imply that morphological features play different roles in retrieval for the two dependencies and that there is not a uniform mapping from grammatical constraints to retrieval cues.

One possibility suggested by Dillon and colleagues is that agreement and reflexives use distinct sets of retrieval cues to access a licensor (Dillon et al., 2013). For example, reflexive licensing might engage the same retrieval mechanism as agreement, but might deploy only structural retrieval cues that target the local subject, implementing morphological constraints as a post-retrieval check. Another option suggested by Cunnings and Sturt is that retrieval for

\footnotetext{
2 The only exception to this pattern was observed in second-pass reading times, where the presence of a gender-matched distractor led to faster reading times at the reflexive, but only in grammatical conditions. However, the direction of the effect was reversed in Experiment 2.
} 
reflexive licensing deploys both structural and morphological cues during retrieval, but preferentially weights structural cues over morphological cues (Cunnings \& Sturt, 2014). A third option is that agreement and reflexives could engage qualitatively different retrieval mechanisms: whereas agreement might engage a cue-based mechanism that deploys a combination of structural and non-structural cues, reflexives might engage a serial, structureguided search that exploits local structural relations to distinguish grammatically relevant licensors from grammatically irrelevant ones (see Dillon, 2014).

Existing accounts differ in how they use morphological cues in retrieval, but they agree that structural information is prioritized over morphological information during retrieval for reflexive licensing, but not for agreement licensing. This conclusion is non-trivial, as it casts doubt upon the general assumption that all linguistic dependencies are uniformly resolved using a single, error-prone retrieval mechanism that deploys a combination of structural and non-structural retrieval cues to target specific items in content-addressable memory, as suggested in previous research (e.g., Martin \& McElree, 2008; McElree, 2000; McElree, Foraker, \& Dyer, 2003; Lewis \& Vasishth, 2005; Lewis, Vasishth, \& Van Dyke, 2006; Van Dyke \& McElree, 2011; for a review, see McElree 2006). If the retrieval cues used to resolve different linguistic dependencies are not straightforwardly predictable from the well-formedness constraints on those dependencies, then it also raises the question of how learners might converge on the retrieval strategies to deploy for each dependency.

\section{Revisiting previous conclusions about attraction}

There are two reasons to revisit previous conclusions about the contrasting retrieval profiles for reflexives and agreement. The first reason is based on recent findings showing that a related class of anaphoric dependencies is susceptible to attraction. Parker and colleagues recently found that emphatic reflexives in adjunct control sentences like (6) are susceptible to attraction, but only selectively (Parker et al., 2015). In (6), the reflexive himself must take the higher subject the doctor / discovery\} as its antecedent.

(6) The \{doctor / discovery\} that the researcher described was certified after debunking the myth himself. 
Results from Experiment 2 of Parker et al. (2015) showed a clear attraction effect at the reflexive when the target subject mismatched the reflexive in gender and animacy, e.g., discovery. However, in Experiment 3, the attraction effect disappeared when the reflexive and target subject mismatched only in gender, e.g., nurse ... himself, replicating previous findings (e.g., Cunnings \& Sturt, 2014; Sturt, 2003; Dillon et al., 2013).

Parker and colleagues suggested that this contrast might reflect the degree of feature match between the target subject and the reflexive, i.e., 2-feature mismatch in Experiment 2 vs. 1feature mismatch in Experiment 3. However, the authors did not explicitly test this hypothesis, and it is unclear how representative these cases are of reflexive licensing more generally. It is important to determine whether the attraction effects reported by Parker and colleagues generalize to a broader set of structural environments and a wider range of anaphoric elements, including direct object reflexives like those tested in previous studies (e.g., Cunnings \& Sturt, 2014; Sturt, 2003; Dillon et al., 2013).

The second reason to revisit previous conclusions about attraction effects is inspired by computational simulations. Building on the findings reported in Parker et al. (2015), we investigated how the degree of feature match between the reflexive and target subject might impact reflexive licensing using a computational model of memory access based on the ACT-R model of sentence comprehension (Lewis \& Vasishth, 2005). We systematically varied the degree of feature match between the target subject and the non-structural retrieval cues at the reflexive (perfect match, 1-feature mismatch, 2-feature mismatch, and 3-feature mismatch) using the sentences from Sturt (2003), e.g., \{She/he\} remembered that the surgeon had pricked herself and The surgeon who treated \{Jennifer/Johnathan\} had pricked herself (the details of the model are provided in the Supplementary Materials).

Figure 1 presents the percentage of the trials when the distractor, rather than the target subject, was retrieved for reflexive licensing in the model simulations. For current purposes, we adopted the common assumption that greater rates of distractor retrieval monotonically correspond to a stronger attraction effect (e.g., Dillon et al., 2013; Kush \& Phillips; 2014; Vasishth et al., 2008). The model predicts a sharp increase in attraction as the number of features that match between the target subject and the reflexive is reduced. This effect is driven by the non-linear "fan" function of the model (Equation 3 in the Supplementary Materials), which 
reduces the strength of association between a cue and the target as a function of the number of items associated with that cue (Anderson, 1974, Anderson \& Reder, 1999).

Figure 1. Percentage of incorrect retrievals for reflexive licensing as a function of probe-totarget similarity as predicted by the ACT-R model (stimuli based on Sturt, 2003).

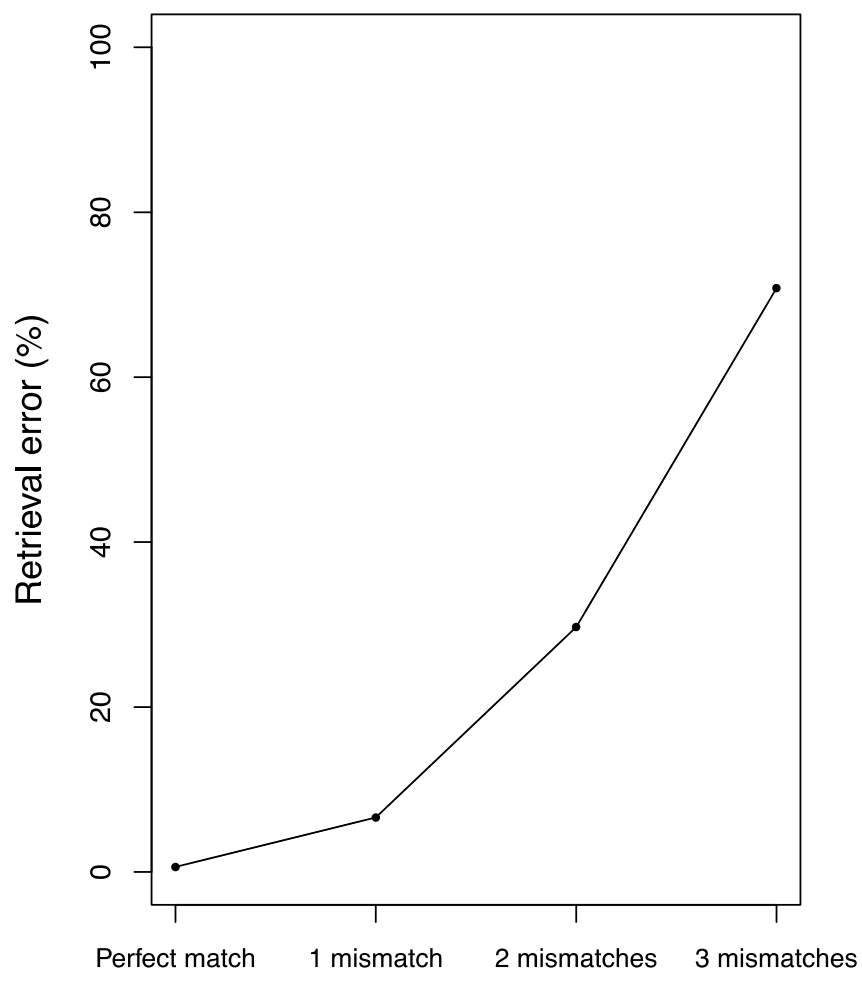

These results imply that previous tests for reflexive attraction may not have used strong enough reflexive-antecedent mismatches to reliably elicit an effect. Previous studies tested contexts in which the illicit reflexive-antecedent dependency involved only a single feature mismatch, e.g., gender (Cunnings \& Sturt, 2014; Sturt, 2003) or number (Dillon et al., 2013). Based on the computational simulations and previous studies (e.g., Parker et al., 2015), we predict that reflexives should be more susceptible to attraction when the reflexive and target subject mismatch in multiple features.

\section{The present study}

The present study tests the prediction that reflexives should be more susceptible to attraction when the reflexive and the correct target subject mismatch in multiple features. Across three eye- 
tracking while reading experiments, we compared contexts where the target subject and a direct object reflexive mismatched in one feature, e.g., gender or number, and in two features, e.g., animacy + gender $($ Experiment 1$)$, animacy + number (Experiment 2$)$, and gender + number (Experiment 3). We also tested additional conditions involving agreement in Experiment 2 to compare the strength of attraction effects with agreement and anaphora.

\section{Experiment 1}

Experiment 1 was designed to test whether direct object reflexives would show attraction effects (i.e., facilitatory interference) in contexts with a stronger reflexive-antecedent feature mismatch than used in most previous studies. We compared contexts where the reflexive and the correct target subject mismatched in gender (1-feature mismatch) and gender and animacy (2-feature mismatch). We chose this feature combination because it has been shown to yield attraction for a related class of anaphors (e.g., Parker et al., 2015).

If attraction effects for reflexives depend on the degree of feature match between the reflexive and target subject, we should observe stronger attraction at the reflexive in the 2-feature mismatch context than in the 1-feature mismatch context. Alternatively, if reflexive licensing exclusively uses structural cues, e.g., local subject, for retrieving an antecedent, as previously claimed (e.g., Dillon et al., 2013), we should not observe attraction, regardless of the degree of mismatch between the reflexive and the target subject.

\section{Participants}

Thirty members of the University of Maryland community participated in Experiment 1. Participants were either compensated $\$ 10$ or received credit in an introductory linguistics course. The eye-tracking experiment, including setup and calibration, lasted approximately 45 minutes.

\section{Stimuli}

We crossed three levels of target match (match/1-feature mismatch/2-feature mismatch) with two levels of distractor match (match/mismatch) to result in a $3 \times 2$ within-participants design. The factor target match was manipulated by varying the degree of match between the target subject and the reflexive, such that they fully matched (match), mismatched in gender only (1-feature 
mismatch), or mismatched in animacy and gender (2-feature mismatch). The factor distractor match was manipulated by varying the gender of the distractor.

Thirty-six item sets of the form shown in Table 1 were constructed. The structure of the items followed that reported in Sturt (2003) Experiment 1. Across all conditions, the distractor appeared in the subject position of the main clause, and the target appeared in the subject position of an embedded complement clause that contained the reflexive in direct object position. Target and distractor NPs used a mix of stereotypical gender, e.g., nurse, and definitional gender, e.g., father from previous studies (e.g., Dillon et al., 2013; Parker et al., 2015; Sturt, 2003). Previous work has shown that stereotypical gender and definitional gender behave similarly with respect to retrieval effects (Kreiner, Sturt, \& Garrod, 2008). The main clause verb was immediately followed by the embedded clause. The embedded verb was always a non-agreeing past tense verb. The lack of agreement reduced the likelihood that subject-verb agreement would provide independent cues for subject retrieval. The verb was followed immediately by a direct object reflexive. The reflexive was followed by a 3-7 word spillover region.

The 36 item sets were combined with 72 grammatical fillers of similar length and complexity, for a total of 108 sentences. Half of the target items and half of the fillers were followed by a comprehension question, which addressed various parts of the target or filler sentence to prevent participants from developing superficial reading strategies that would allow them to answer the question without reading the entire sentence. The entire set of stimuli can be found in the Supplemental Materials.

Table 1. Sample set of items for Experiment 1. Pre-critical, critical, and spillover regions included in the analysis are indicated by slashes.

\section{Distractor match}

The strict librarian said that/ the studious schoolgirl reminded/ herself/ about the/ overdue book.

\section{Distractor mismatch}

The strict father said that/ the studious schoolgirl reminded/ herself/ about the/ overdue book.

\section{Distractor match}

$\perp$ The strict librarian said that/ the studious schoolboy reminded/ herself/ about the/

巳 


\section{Distractor mismatch}

The strict father said that/ the studious schoolboy reminded/ herself/ about the/ overdue book.

\section{Distractor match}

The strict librarian said that/ the brief memo reminded/ herself/ about the/ overdue book.

\section{Distractor mismatch}

The strict father said that/ the brief memo reminded/ herself/ about the/ overdue book.

\section{Procedure}

The 36 item sets were distributed across 6 lists in a Latin Square design, and 6 participants were assigned to each list. Each list was randomized along with the filler sentences. Sentences were presented in 12-point Courier font. All sentences in the experiment fit one line on the visual display. Eye movements were recorded using an EyeLink 1000 tower-mount eye-tracker, which sampled at $1000 \mathrm{~Hz}$. Participants had binocular vision during recording, but only the right eye was tracked. The tower was 32 inches from the visual display giving participants approximately 5 characters per degree of visual angle.

Prior to the experiment, participants were familiarized with the eye-tracking setup and were given four practice trials. While seated at the tower-mount, participants' heads were immobilized using a chin rest and a forehead restraint that was adjusted by height for comfort. Before the experiment, and whenever necessary throughout the experiment, the experimenter calibrated the eye-tracker using a 9-point display procedure to ensure accurate recording of eye-movements. Participants began each trial by fixating on a marker at the beginning of the sentence, which triggered the display of the entire sentence. Participants ended the presentation sentences by pressing a button on a hand-held controller, which triggered the presentation of a comprehension question on trials that had a comprehension question or transitioned to the next trial on trials that did not. Participants were allowed to take breaks as often as necessary throughout the experiment. The experimenter recalibrated the eye-tracker following each break.

\section{Data analysis}


Sentences were divided into five regions following Sturt (2003), as indicated in Table 1. We report means and statistical analyses from the pre-critical, critical, and spillover regions. The precritical region consisted of the words between the complementizer that and the reflexive (exclusive). The reflexive region consisted of the reflexive anaphor herself. The spillover region consisted of the two words following the reflexive. The average skipping rate for the critical regions was $8 \%$.

For each region of interest, we report four measures: first-pass reading time, right-bound (gopast) reading time, regression path duration, and second pass reading time. First pass reading time is the sum of all fixations in a region before it is exited, either to the left or right. Rightbound reading time is the sum of all fixations in a region before it is exited to the right. Regression path duration is the sum of fixations in a region and all the regions to the left, starting when the region was entered until it was exited to the right. Second pass reading time is the sum of fixations in a region after the first pass.

Statistical analyses were carried out for each measure and region of interest using linear mixed effects models provided by the lme 4 package (Bates, Maechler, Bolker, \& Walker, 2014) in the R software environment (R Development Core Team, 2014). We first ran a model to test for a main effect of target match ('grammaticality'), a main effect of distractor match, and the interaction between the two main effects. Treatment coding was applied using the level match of the factor target match as the baseline, and the level mismatch of the factor distractor match as the baseline. These baselines were chosen as they correspond to conditions where there is no effect of grammaticality and no attraction effect. To maximize the chances of observing an attraction effect, we also performed pairwise comparisons within the ungrammatical 1-feature mismatch and 2-feature mismatch conditions separately (labeled as 1-feature attraction and 2feature attraction in the model coefficient tables). We also report an interaction term that compares the size of the attraction effect across the 1- vs. 2-feature mismatch conditions. All models were fit with a full variance-covariance matrix, i.e., a maximal random effects structure with random intercepts by participants and items and random slopes for all fixed effects and their interaction (Baayen, Davidson, \& Bates, 2008; Barr, Levy, Scheepers, \& Tily, 2013). If the model failed to converge or the variance-covariance matrix was degenerate (e.g., correlations were close to \pm 1 ), random slopes for items or participants were removed. A fixed effect was considered significant if its absolute $t$-value was greater than 2 , which indicates that its $95 \%$ 
confidence interval did not include 0 (Gelman \& Hill, 2007). To reduce non-normality, statistical analyses were carried out over log-transformed reading times (Box \& Cox, 1964). Missing observations contributed a value of $0 \mathrm{~ms}$ to the cell mean, following previous studies (e.g., Kush et al, 2015; Sturt, 2003).

\section{Results}

Table 2 provides the mean raw reading times by measure and region. Table 3 provides a summary of the statistical analyses. Figure 2 provides a plot of the mean raw reading times by measure at the critical reflexive region.

No significant effects or interactions were observed in first-pass reading times or right-bound reading times in the pre-critical, critical, or spillover regions. A main effect of grammaticality was observed in second-pass reading times at the critical reflexive region for both the 1- and 2feature mismatch conditions. This effect was driven by longer reading times in the ungrammatical mismatch conditions relative to the grammatical match conditions. The effect also reached significance in the pre-critical region for the 1-feature mismatch condition, indicating that the processing disruption associated with the target feature mismatch influenced re-reading of both the reflexive region and the previous region.

Importantly, for the 1-feature mismatch conditions, there was no interaction of grammaticality with distractor match nor an effect of attraction in any region or measure, replicating previous findings (e.g., Dillon et al., 2013; Sturt, 2003). However, for the 2-feature mismatch conditions, there was a significant interaction of grammaticality with distractor match at the reflexive in second-pass reading times, driven by attraction in second-pass reading times. This effect manifested as faster reading times for ungrammatical sentences with a feature matching distractor relative to ungrammatical sentences with a feature mismatching distractor. Regression path durations at the reflexive for the 2-feature mismatch conditions also showed a significant effect of attraction in raw reading times $(t>2)$. However, this effect did not reach significance with the log-transformed values, suggesting that the difference in raw reading times is driven by extreme values, which were normalized by the log-transformation.

Table 2. Table of means in Experiment 1. Standard error by participants is shown in parentheses.

\begin{tabular}{lll} 
& Regions & \\
\hline Pre-critical & Critical & Spillover \\
\hline
\end{tabular}




\begin{tabular}{|c|c|c|c|}
\hline \multicolumn{4}{|l|}{ First-pass reading time } \\
\hline Target match, distractor match & $1052(47)$ & $215(12)$ & $227(17)$ \\
\hline Target match, distractor mismatch & $1020(48)$ & $205(10)$ & $235(16)$ \\
\hline Target 1-feature mismatch, distractor match & $924(39)$ & $229(13)$ & $261(20)$ \\
\hline Target 1-feature mismatch, distractor mismatch & $960(37)$ & $243(14)$ & $274(23)$ \\
\hline Target 2-feature mismatch, distractor match & $873(37)$ & $216(11)$ & $202(15)$ \\
\hline Target 2-feature mismatch, distractor mismatch & $934(48)$ & $226(12)$ & $221(19)$ \\
\hline \multicolumn{4}{|l|}{$\underline{\text { Right-bound reading time }}$} \\
\hline Target match, distractor match & $1207(46)$ & $225(13)$ & $249(21)$ \\
\hline Target match, distractor mismatch & $1180(47)$ & $218(11)$ & $262(19)$ \\
\hline Target 1-feature mismatch, distractor match & $1132(37)$ & $256(15)$ & $327(28)$ \\
\hline Target 1-feature mismatch, distractor mismatch & $1088(35)$ & $276(17)$ & $337(30)$ \\
\hline Target 2-feature mismatch, distractor match & $1020(38)$ & $228(12)$ & $242(18)$ \\
\hline Target 2-feature mismatch, distractor mismatch & $1091(44)$ & $258(16)$ & $284(26)$ \\
\hline \multicolumn{4}{|l|}{$\underline{\text { Regression path duration }}$} \\
\hline Target match, distractor match & $1265(50)$ & $274(21)$ & $314(38)$ \\
\hline Target match, distractor mismatch & $1250(53)$ & $260(17)$ & $403(55)$ \\
\hline Target 1-feature mismatch, distractor match & $1211(43)$ & $318(27)$ & $486(59)$ \\
\hline Target 1-feature mismatch, distractor mismatch & $1139(39)$ & $373(37)$ & $539(65)$ \\
\hline Target 2-feature mismatch, distractor match & $1081(42)$ & $273(18)$ & $329(34)$ \\
\hline Target 2-feature mismatch, distractor mismatch & $1189(51)$ & $365(44)$ & $453(60)$ \\
\hline \multicolumn{4}{|l|}{$\underline{\text { Second-pass reading time }}$} \\
\hline Target match, distractor match & $761(78)$ & $193(22)$ & $223(26)$ \\
\hline Target match, distractor mismatch & $750(66)$ & $196(21)$ & $254(25)$ \\
\hline Target 1-feature mismatch, distractor match & $1027(93)$ & $338(30)$ & $368(33)$ \\
\hline Target 1-feature mismatch, distractor mismatch & $1005(80)$ & $314(27)$ & $336(34)$ \\
\hline Target 2-feature mismatch, distractor match & $561(54)$ & $169(18)$ & $248(24)$ \\
\hline Target 2-feature mismatch, distractor mismatch & $749(65)$ & $350(34)$ & $352(38)$ \\
\hline
\end{tabular}

Table 3. Summary of statistical analyses by region and measure in Experiment 1. Significant coefficients $(|t|>2)$ are in bold.

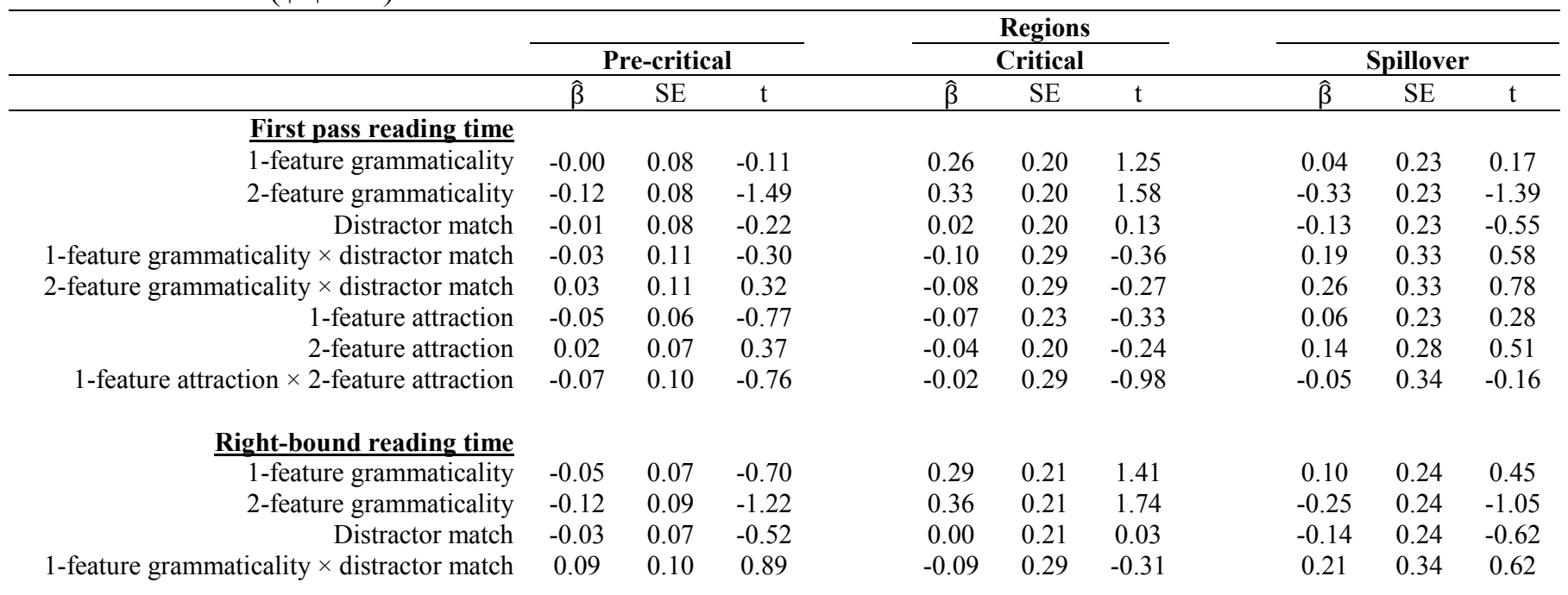




\begin{tabular}{|c|c|c|c|c|c|c|c|c|c|}
\hline 2-feature grammaticality $\times$ distractor match & 0.04 & 0.10 & 0.41 & -0.09 & 0.29 & -0.32 & 0.25 & 0.34 & 0.73 \\
\hline 1-feature attraction & 0.05 & 0.05 & -1.10 & -0.08 & 0.22 & -0.38 & 0.06 & 0.24 & 0.27 \\
\hline 2-feature attraction & 0.00 & 0.06 & 0.04 & -0.08 & 0.20 & -0.42 & 0.11 & 0.29 & 0.39 \\
\hline 1-feature attraction $\times 2$-feature attraction & 0.06 & 0.08 & 0.72 & 0.00 & 0.30 & 0.00 & 0.00 & 0.36 & -0.02 \\
\hline \multicolumn{10}{|l|}{$\underline{\text { Regression path duration }}$} \\
\hline 1-feature grammaticality & -0.05 & 0.06 & -0.86 & 0.33 & 0.21 & 1.54 & 0.17 & 0.25 & 0.68 \\
\hline 2-feature grammaticality & -0.07 & 0.06 & -1.09 & 0.40 & 0.22 & 1.83 & -0.22 & 0.25 & -0.88 \\
\hline Distractor match & -0.04 & 0.06 & -0.59 & 0.00 & 0.21 & 0.03 & -0.18 & 0.25 & -0.72 \\
\hline 1-feature grammaticality $\times$ distractor match & 0.11 & 0.09 & 1.14 & -0.13 & 0.31 & -0.43 & 0.24 & 0.35 & 0.67 \\
\hline 2-feature grammaticality $\times$ distractor match & 0.01 & 0.09 & 0.10 & -0.12 & 0.31 & 0.41 & 0.26 & 0.36 & 0.72 \\
\hline 1-feature attraction & 0.07 & 0.05 & -1.37 & -0.12 & 0.23 & -0.55 & 0.05 & 0.25 & 0.21 \\
\hline 2-feature attraction & -0.01 & 0.06 & -0.24 & -0.11 & 0.21 & -0.54 & 0.08 & 0.30 & 0.29 \\
\hline 1-feature attraction $\times 2$-feature attraction & 0.9 & 0.08 & 1.11 & -0.01 & 0.31 & -0.33 & -0.02 & 0.34 & -0.06 \\
\hline \multicolumn{10}{|l|}{$\underline{\text { Second-pass reading time }}$} \\
\hline 1-feature grammaticality & 0.59 & 0.29 & 2.04 & 1.05 & 0.29 & 3.51 & 0.43 & 0.26 & 1.64 \\
\hline 2-feature grammaticality & -0.11 & 0.27 & -0.41 & 0.89 & 0.31 & 2.84 & 0.47 & 0.26 & 1.77 \\
\hline Distractor match & -0.29 & 0.31 & -0.92 & -0.01 & 0.30 & -0.04 & -0.05 & 0.26 & -0.18 \\
\hline 1-feature grammaticality $\times$ distractor match & 0.11 & 0.40 & 0.28 & -0.02 & 0.43 & -0.05 & 0.35 & 0.37 & 0.93 \\
\hline 2-feature grammaticality $\times$ distractor match & -0.37 & 0.48 & 0.77 & -0.92 & 0.42 & -2.16 & 0.05 & 0.38 & 0.15 \\
\hline 1-feature attraction & -0.17 & 0.25 & -0.69 & -0.04 & 0.26 & -0.16 & 0.30 & 0.26 & 1.14 \\
\hline 2-feature attraction & -0.65 & 0.37 & -1.75 & -0.93 & 0.27 & -3.41 & 0.03 & 0.33 & 0.10 \\
\hline 1 -feature attraction $\times 2$-feature attraction & 0.48 & 0.38 & 1.24 & 0.89 & 0.38 & 2.33 & 0.29 & 0.38 & 0.77 \\
\hline
\end{tabular}

Figure 2. Mean raw reading times by measure at the critical reflexive region in Experiment 1. Error bars indicate standard error of the mean. 

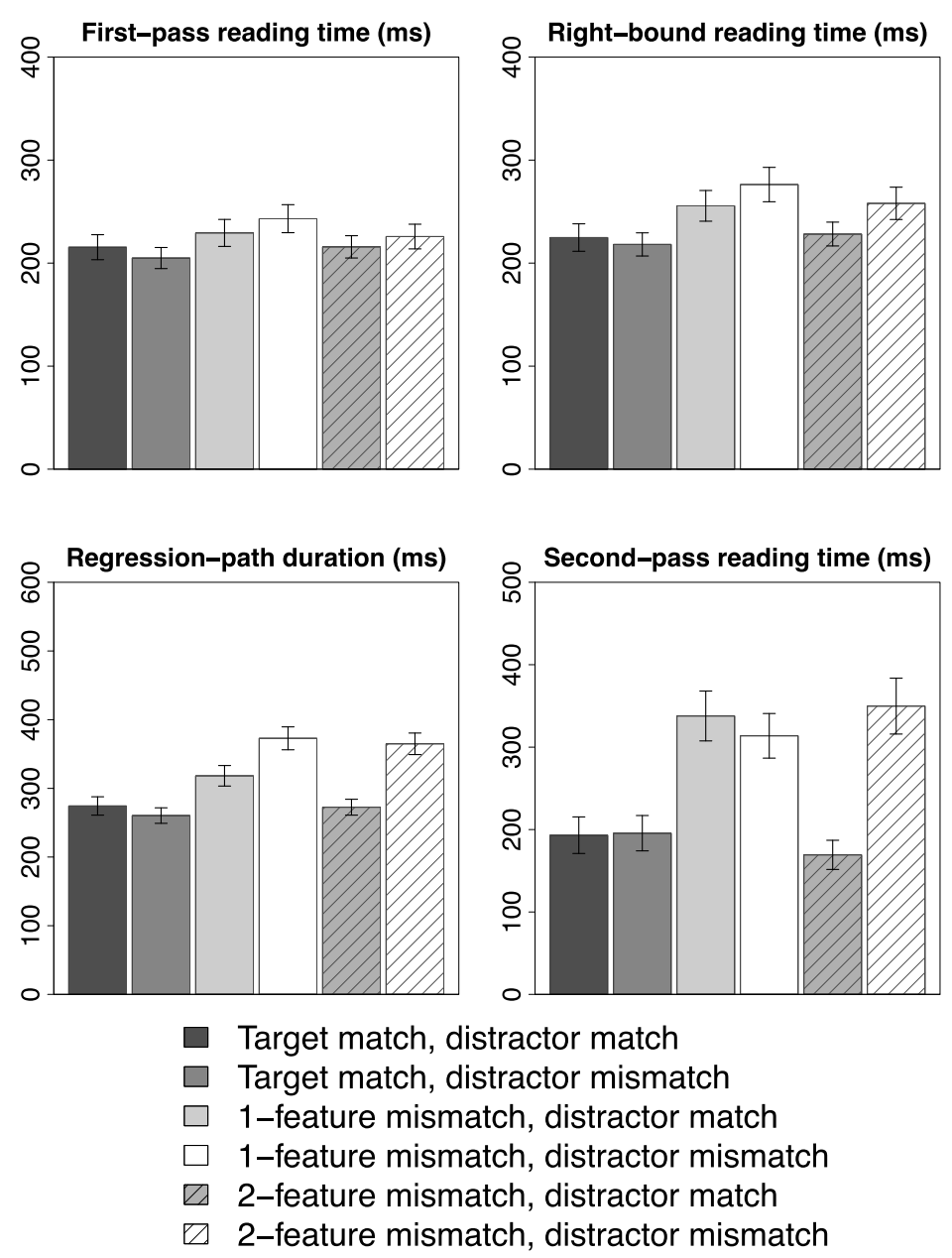

\section{Discussion}

Experiment 1 tested the prediction that reflexives should be more susceptible to attraction in contexts with a stronger reflexive-antecedent feature mismatch. When the reflexive mismatched the target in gender, e.g., schoolgirl ... herself, no attraction effects were observed, replicating previous findings (e.g., Cunnings \& Sturt, 2014; Sturt, 2003, Dillon et al., 2013). By contrast, when the reflexive mismatched the target subject in both gender and animacy, e.g., memo ... herself, we observed a clear attraction effect.

These results demonstrate that reflexives are indeed susceptible to attraction, but only selectively: by making a minimal change to the strength of the feature match between the target subject and reflexive, we were able to systematically induce reflexive attraction effects. However, since such effects are rarely observed for reflexives, it is important to verify that they 
generalize across licensing configurations and feature combinations. We address this issue in Experiment 2.

\section{Experiment 2}

Experiment 2 had two goals. The first goal was to test whether reflexive attraction would extend to other feature combinations and licensing configurations. To this end, we tested contexts where the reflexive and target subject mismatched in number and animacy, as opposed to gender and animacy. We manipulated number alongside animacy because previous research has shown that reflexives resist attraction in the presence of a number mismatch with the target (e.g., Dillon et al., 2013). We also reversed the relative linear positions of the target and distractor following Sturt (2003), which allowed us to test whether reflexive attraction would be robust across licensing configurations. The second goal was to obtain a within-subjects comparison of attraction effects for reflexives and agreement. To achieve this, we replaced the target vs. 1feature mismatch comparison with maximally similar conditions involving subject verb agreement. As such, Experiment 2 was not designed to compare 1- vs. 2-feature mismatches for reflexives.

\section{Participants}

Thirty members of the University of Maryland community participated in Experiment 2. Participants were either compensated $\$ 10$ or received credit in an introductory linguistics course. The eye-tracking experiment, including setup and calibration, lasted approximately 45 minutes.

\section{Stimuli}

We manipulated target match (match/1-feature mismatch/2-feature mismatch) and distractor match (match/mismatch). However, unlike in Experiment 1, the target match and 1-feature mismatch conditions involved subject-verb agreement. Across all items, the target subject was always singular and inanimate. In the target match conditions, the agreeing verb was singular, e.g., was, and matched the number of the target subject. In the 1-feature mismatch conditions, the agreeing verb was plural, e.g., were, and mismatched the target subject only in number. In the 2feature mismatch conditions, the reflexive was plural, e.g., themselves, and mismatched the target subject in both number and animacy. 
The linear positions of the target subject and distractor were reversed relative to Experiment 1, following Sturt (2003; Experiment 2). In all conditions, the target subject was modified by an object relative clause that contained the distractor in subject position. The relative clause never overtly expressed agreement to prevent attraction before the critical region. In the agreement conditions, the main clause verb phrase consisted of the critical agreeing auxiliary verb (was or were) immediately followed by the main verb. In the reflexive conditions, the main clause verb was always a non-agreeing past tense verb followed immediately by a direct object reflexive.

Thirty-six item sets of the form shown in Table 4 were constructed. The 36 item sets were mixed with 72 grammatical fillers of similar length and complexity, for a total of 108 sentences. Half of the target items and half of the fillers were followed by a comprehension question.

Table 4. Sample set of items for Experiment 2. Pre-critical, critical, and spillover regions included in the analysis are indicated by slashes.

\begin{tabular}{|c|c|c|}
\hline & 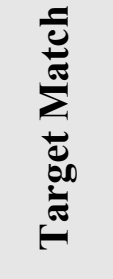 & $\begin{array}{l}\text { Distractor match } \\
\text { The soothing tea that/ the nervous student drank/ was imported/ from India./ } \\
\text { Distractor mismatch } \\
\text { The soothing tea that/ the nervous students drank/ was imported/ from India./ }\end{array}$ \\
\hline$\frac{000}{4}$ & 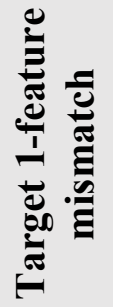 & $\begin{array}{l}\text { Distractor match } \\
\text { The soothing tea that/ the nervous students drank/ were imported/ from India./ } \\
\text { Distractor mismatch } \\
\text { The soothing tea that/ the nervous student drank/ were imported/ from India./ }\end{array}$ \\
\hline 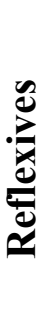 & 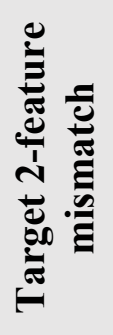 & $\begin{array}{l}\text { Distractor match } \\
\text { The soothing tea that/ the nervous students drank calmed/ themselves/ down after/ } \\
\text { the test. } \\
\text { Distractor mismatch } \\
\text { The soothing tea that/ the nervous student drank calmed/ themselves/ down after/ } \\
\text { the test. }\end{array}$ \\
\hline
\end{tabular}

Data analysis 
Data analysis proceeded as in Experiment 1. However, we did not analyze the effect of grammaticality (target match) for the reflexive 2-feature mismatch conditions. This effect is not interpretable since it involves comparing grammatical and ungrammatical sentences at different critical words for different dependencies (verb vs. reflexive). Regioning for the agreement conditions followed the same regioning used for the agreement conditions in Dillon et al. (2013). The average skipping rate for the critical regions was $2 \%$.

\section{Results}

Table 5 provides the mean raw reading times by measure and region. Table 6 provides a summary of the statistical analyses. Figure 3 provides a plot of the mean raw reading times by measure at the critical reflexive region.

No significant effects or interactions were observed in first-pass, right-bound reading times, or regression path duration in the pre-critical region. Agreement conditions showed a significant effect of grammaticality across all measures at the critical region, which was driven by longer reading times in the 1-feature mismatch conditions relative to the match conditions. This effect also reached significance in second-pass reading times in the pre-critical region, suggesting that the processing disruption in the target mismatch conditions influenced re-reading of the previous region. Crucially, there was a significant interaction of grammaticality with distractor match in second pass reading times at the critical region. This effect was driven by attraction in the ungrammatical mismatch conditions. Reflexive conditions showed a significant effect of attraction in first-pass, right-bound, and second-pass reading times at the critical region and spillover region.

Table 5. Table of means in Experiment 2. Standard error by participants is shown in parentheses.

\begin{tabular}{rccc}
\hline First-pass reading time & \multicolumn{3}{c}{ Regions } \\
\cline { 3 - 4 } Target match, distractor match & $1109(42)$ & Critical & Spillover \\
\hline Target match, distractor mismatch & $1077(41)$ & $337(15)$ & $652(35)$ \\
Target 1-feature mismatch, distractor match & $1089(40)$ & $336(14)$ & $656(31)$ \\
Target 1-feature mismatch, distractor mismatch & $1068(46)$ & $409(18)$ & $662(36)$ \\
Target 2-feature mismatch, distractor match & $1441(58)$ & $231(9)$ & $754(35)$ \\
Target 2-feature mismatch, distractor mismatch & $1449(59)$ & $277(11)$ & $711(33)$
\end{tabular}

Right-bound reading time 


\section{Target match, distractor match}

Target match, distractor mismatch

Target 1-feature mismatch, distractor match

Target 1-feature mismatch, distractor mismatch

Target 2-feature mismatch, distractor match

Target 2-feature mismatch, distractor mismatch

\section{Regression path duration}

Target match, distractor match

Target match, distractor mismatch

Target 1-feature mismatch, distractor match

Target 1-feature mismatch, distractor mismatch

Target 2-feature mismatch, distractor match

Target 2-feature mismatch, distractor mismatch

$\begin{array}{llc}1257(45) & 375(17) & 938(44) \\ 1194(45) & 373(16) & 926(43) \\ 1249(42) & 479(25) & 1010(53) \\ 1203(43) & 485(22) & 1040(53) \\ 1728(76) & 254(12) & 1057(45) \\ 1725(71) & 302(12) & 1146(47)\end{array}$

$1322(58)$

$473(40)$

1938 (127)

$1272(57)$

$419(22)$

$1871(153)$

1309 (49)

$613(50)$

$2232(151)$

$1270(46)$

$696(56)$

2369 (166)

1853 (106)

357 (32)

2295 (151)

1847 (98)

$401(36)$

Second-pass reading time

Target match, distractor match

Target match, distractor mismatch

Target 1-feature mismatch, distractor match

Target 1-feature mismatch, distractor mismatch

Target 2-feature mismatch, distractor match

Target 2-feature mismatch, distractor mismatch

$\begin{array}{ccc}669(75) & 256(25) & 286(36) \\ 576(69) & 212(26) & 270(34) \\ 788(73) & 328(35) & 348(45) \\ 843(82) & 395(35) & 378(43) \\ 1021(110) & 147(19) & 304(39) \\ 1182(113) & 261(22) & 438(44)\end{array}$

Table 6. Summary of statistical analyses by region and measure in Experiment 2. Significant coefficients $(|t|>2)$ are in bold.

\begin{tabular}{|c|c|c|c|c|c|c|c|c|c|}
\hline & \multicolumn{3}{|c|}{ Pre-critical } & \multicolumn{3}{|c|}{$\begin{array}{l}\text { Regions } \\
\text { Critical } \\
\end{array}$} & \multicolumn{3}{|c|}{ Spillover } \\
\hline \multicolumn{10}{|l|}{ First pass reading time } \\
\hline Distractor match & 0.02 & 0.06 & 0.43 & -0.01 & 0.11 & -0.12 & 0.01 & 0.08 & 0.21 \\
\hline Agreement grammaticality $\times$ distractor match & -0.00 & 0.09 & -0.02 & -0.09 & 0.16 & -0.60 & -0.09 & 0.12 & -0.76 \\
\hline Agreement attraction & 0.02 & 0.09 & 0.26 & -0.11 & 0.10 & -1.03 & -0.07 & 0.08 & -0.80 \\
\hline \multicolumn{10}{|l|}{$\underline{\text { Right-bound reading time }}$} \\
\hline$\overline{\text { Agreement grammaticality }}$ & 0.00 & 0.04 & 0.11 & 0.38 & 0.11 & 3.32 & 0.14 & 0.08 & 1.79 \\
\hline Distractor match & 0.05 & 0.04 & 1.22 & -0.00 & 0.11 & -0.08 & 0.05 & 0.08 & 0.66 \\
\hline Agreement grammaticality $\times$ distractor match & -0.03 & 0.06 & -0.51 & -0.09 & 0.16 & -0.56 & -0.19 & 0.11 & -1.68 \\
\hline Agreement attraction & 0.01 & 0.05 & 0.30 & -0.10 & 0.11 & -0.93 & 0.13 & 0.07 & -1.81 \\
\hline Reflexive attraction & -0.01 & 0.03 & -0.28 & -0.29 & 0.12 & -2.36 & -0.09 & 0.08 & -1.07 \\
\hline \multicolumn{10}{|l|}{ Regression path duration } \\
\hline Distractor match & 0.04 & 0.04 & 0.94 & 0.02 & 0.12 & 0.19 & 0.11 & 0.09 & 1.11 \\
\hline Agreement grammaticality $\times$ distractor match & -0.04 & 0.06 & -0.64 & -0.18 & 0.17 & -1.06 & -0.25 & 0.14 & -1.84 \\
\hline Agreement attraction & -0.00 & 0.05 & -0.05 & -0.17 & 0.11 & -1.47 & -0.14 & 0.09 & -1.46 \\
\hline Reflexive attraction & -0.00 & 0.03 & -0.15 & -0.25 & 0.13 & -1.90 & -0.20 & 0.10 & -1.87 \\
\hline \multicolumn{10}{|l|}{ Second-pass reading time } \\
\hline$\overline{\text { Agreement grammaticality }}$ & 1.03 & 0.32 & 3.20 & 1.23 & 0.29 & 4.24 & 0.55 & 0.28 & 1.94 \\
\hline Distractor match & 0.36 & 0.34 & 1.04 & 0.44 & 0.28 & 1.56 & -0.00 & 0.28 & -0.01 \\
\hline Agreement grammaticality $\times$ distractor match & -0.57 & 0.43 & -1.30 & -1.16 & 0.43 & -2.68 & -0.37 & 0.40 & -0.92 \\
\hline Agreement attraction & -0.21 & 0.32 & -0.67 & -0.73 & 0.34 & -2.13 & -0.38 & 0.33 & -1.15 \\
\hline
\end{tabular}


Figure 3. Mean raw reading times by measure at the critical region in Experiment 2. Error bars indicate standard error of the mean.
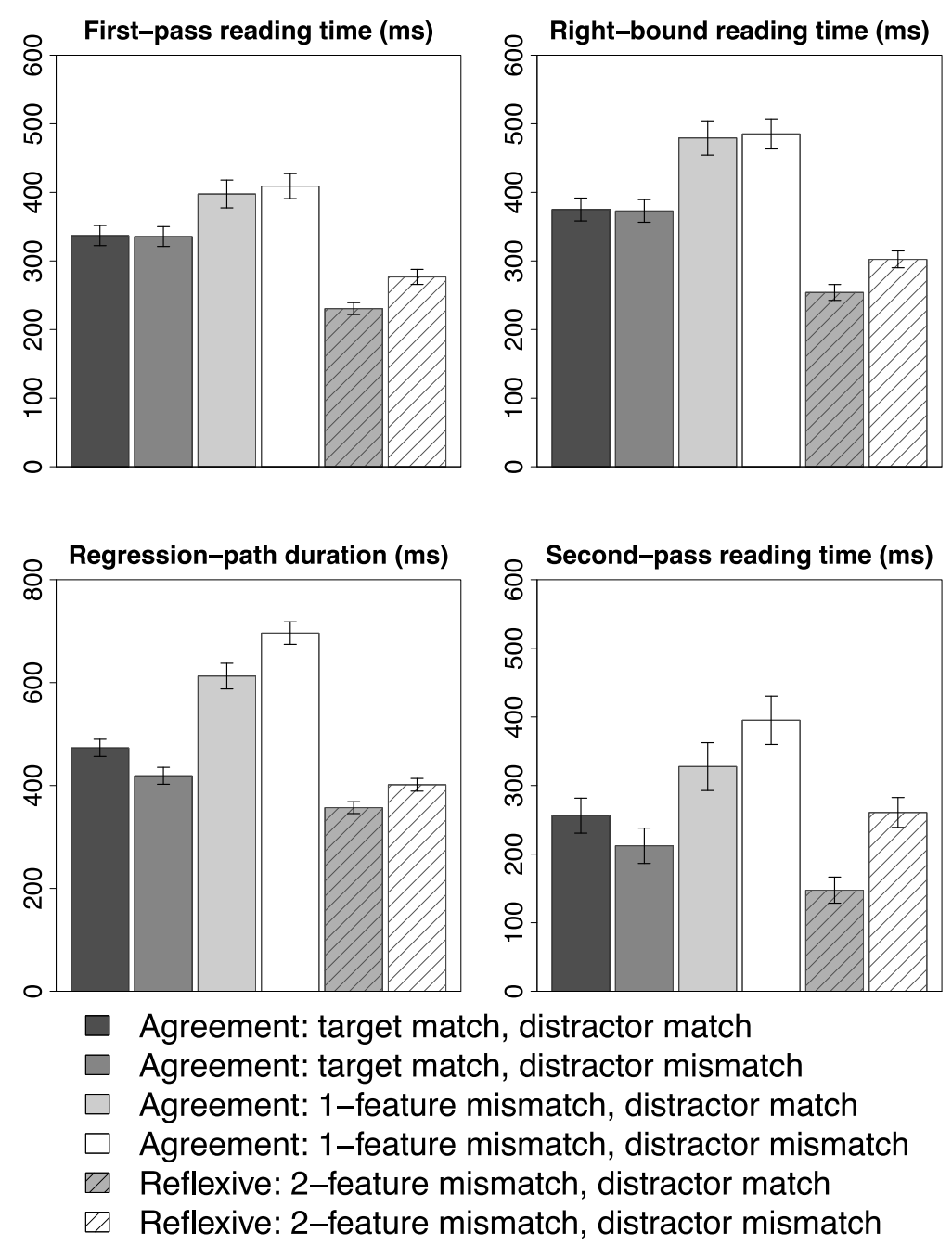

\section{Discussion}

Experiment 2 was designed to achieve two goals. The first goal was to test whether reflexive attraction would extend to other feature combinations and licensing configurations. The second goal was to obtain a within-subjects comparison of attraction effects for reflexives and agreement. The key finding from Experiment 2 is that attraction effects for reflexives are as strong as those observed for agreement, and that reflexive attraction does in fact extend to other feature combinations and licensing configurations. 
There are two concerns about the results of Experiment 2. First, agreement attraction and reflexive attraction showed different timing profiles: reflexives showed attraction across both early and late measures, e.g., first-pass and second-pass reading times, whereas agreement showed attraction only in late measures, e.g., second-pass reading times. However, it is unclear what to make of this contrast, since reflexive attraction was observed only in late measures in Experiment 1. We return to this issue in the General Discussion.

The second concern is that reflexive attraction might be driven by animacy, rather than by the more general property of reflexive-antecedent match. So far, both demonstrations of the reflexive attraction effect in Experiments 1 and 2 have used animacy in combination with other features. These findings are consistent with the view that animacy information has a privileged role in encoding and accessing linguistic items in memory (e.g., Nairne et al., 2013; Van Arsdall et al., 2013). If reflexive attraction is truly a consequence of the strength of the reflexive-antecedent feature match as hypothesized, then we should observe a similar modulation of the effect using a feature combination that does not rely on animacy. We test this possibility in Experiment 3.

\section{Experiment 3}

Experiment 3 was designed to test whether reflexive attraction is driven by the use of animacy features in retrieval or the more general property of reflexive-antecedent match. To achieve this, we held constant the animacy match between the potential licensors and the reflexive, and tested whether a feature combination involving gender and number would trigger the same modulation of the attraction effect seen in Experiment 1. If reflexive attraction is driven by animacy, we expect to see no difference between 1- and 2-feature mismatch contexts. However, if reflective attraction reflects the strength of the reflexive-antecedent match, then we expect to observe the same modulation of attraction effects seen in Experiment 1.

\section{Participants}

Twenty-four members of the University of Maryland community participated in Experiment 3 Participants were either compensated $\$ 10$ or received credit in an introductory linguistics course. The eye-tracking experiment, including setup and calibration, lasted approximately 45 minutes.

\section{Stimuli}


Thirty-six item sets of the form shown in Table 7 were constructed. The experimental stimuli and fillers followed the same design as in Experiment 1, with the exception that in the 2-feature mismatch context, the target subject and reflexive mismatched in gender and number.

Table 7. Sample set of items for Experiment 3. Pre-critical, critical, and spillover regions included in the analysis are indicated by slashes.

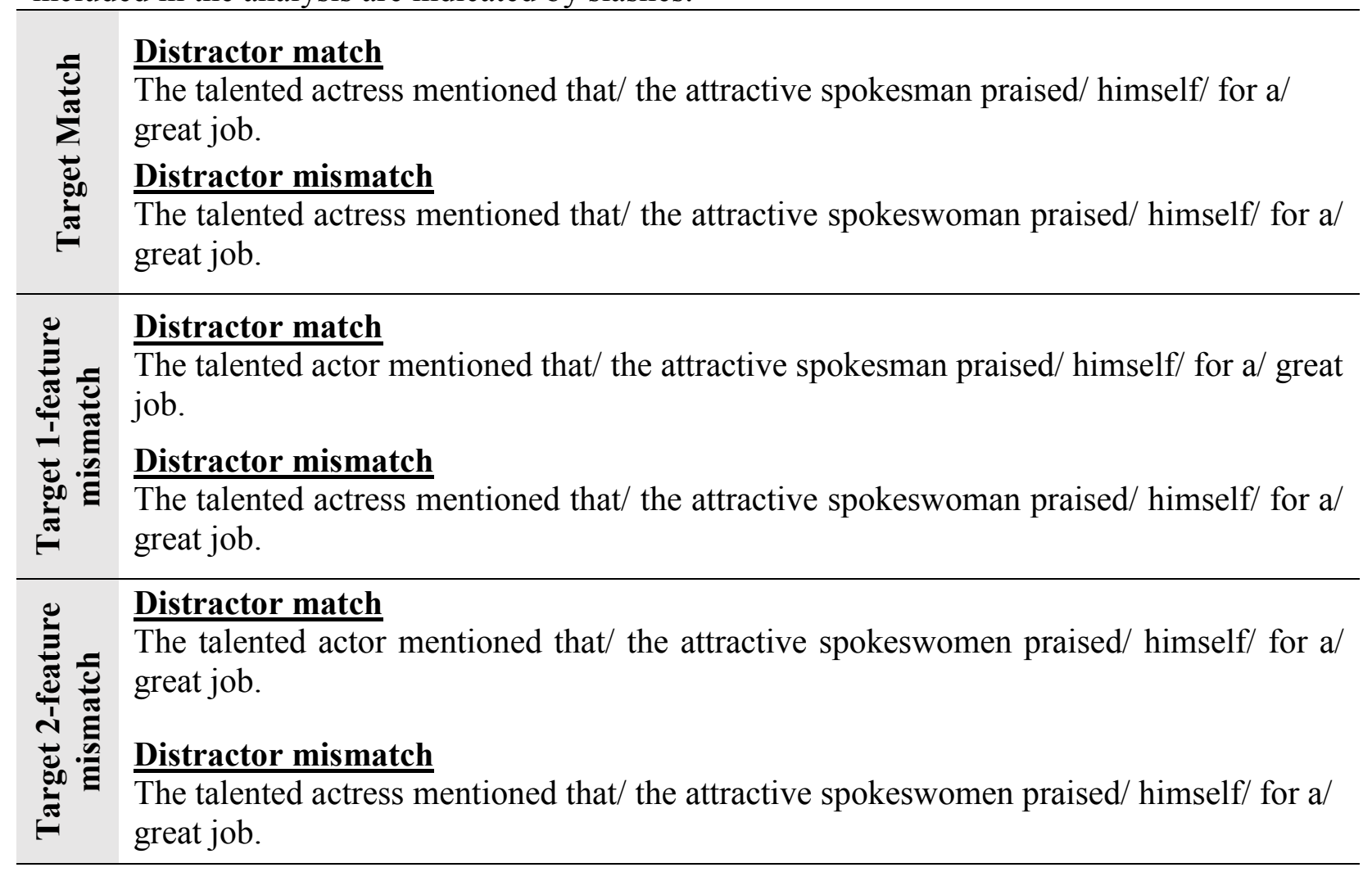

\section{Data analysis}

Data analysis proceeded as in Experiments 1 and 2. The average skipping rate for the critical regions was $5 \%$.

\section{Results}

Table 8 provides the mean raw reading times by measure and region. Table 9 provides a summary of the statistical analyses. Figure 4 provides a plot of the mean raw reading times by measure at the critical reflexive region.

No significant effects or interactions were observed in the pre-critical region in first-pass reading times, right-bound reading times, or regression path durations. A main effect of 
grammaticality was observed in second-pass reading times at the critical region for both the 1and 2-feature mismatch conditions. This effect was carried by longer reading times in the mismatch conditions relative to the match conditions. The effect of grammaticality for the 2feature comparison also reached significance in the pre-critical region. A significant effect of attraction was observed across all measures at the critical region for the 2-feature mismatch conditions. There was a significant interaction of grammaticality with distractor match in the 2feature mismatch conditions in regression path durations and second-pass reading times at the critical region. This effect was driven by attraction in the ungrammatical mismatch conditions. The interaction also reached significance in the pre-critical region. Crucially, for the 1-feature mismatch conditions, there was no interaction of grammaticality with distractor match nor an effect of attraction in any region or measure, replicating previous findings (e.g., Cunnings \& Sturt, 2014; Sturt, 2003; Dillon et al., 2013).

Table 8. Table of means in Experiment 3. Standard error by participants is shown in parentheses.

\begin{tabular}{|c|c|c|c|}
\hline & \multicolumn{3}{|c|}{ Regions } \\
\hline & Pre-critical & Critical & Spillover \\
\hline \multicolumn{4}{|l|}{ First-pass reading time } \\
\hline Target match, distractor match & $947(50)$ & $197(11)$ & $166(13)$ \\
\hline Target match, distractor mismatch & $928(43)$ & $223(12)$ & $155(15)$ \\
\hline Target 1-feature mismatch, distractor match & $805(34)$ & $225(13)$ & $166(23)$ \\
\hline Target 1-feature mismatch, distractor mismatch & $903(40)$ & $223(12)$ & $165(16)$ \\
\hline Target 2-feature mismatch, distractor match & $913(36)$ & $185(12)$ & $156(14)$ \\
\hline Target 2-feature mismatch, distractor mismatch & $882(37)$ & $290(18)$ & $129(14)$ \\
\hline \multicolumn{4}{|l|}{$\underline{\text { Right-bound reading time }}$} \\
\hline Target match, distractor match & $1162(54)$ & $200(11)$ & $196(17)$ \\
\hline Target match, distractor mismatch & $1108(41)$ & $228(12)$ & $185(23)$ \\
\hline Target 1-feature mismatch, distractor match & $1025(36)$ & $245(14)$ & $215(31)$ \\
\hline Target 1-feature mismatch, distractor mismatch & $1088(39)$ & $234(13)$ & $195(20)$ \\
\hline Target 2-feature mismatch, distractor match & $1117(39)$ & $191(13)$ & $191(20)$ \\
\hline Target 2-feature mismatch, distractor mismatch & $1062(40)$ & $329(21)$ & $178(23)$ \\
\hline \multicolumn{4}{|l|}{$\underline{\text { Regression path duration }}$} \\
\hline Target match, distractor match & $1295(79)$ & $221(16)$ & $312(43)$ \\
\hline Target match, distractor mismatch & $1203(57)$ & $251(14)$ & $282(44)$ \\
\hline Target 1-feature mismatch, distractor match & $1199(65)$ & $304(29)$ & $339(54)$ \\
\hline Target 1-feature mismatch, distractor mismatch & $1206(58)$ & $317(40)$ & $342(67)$ \\
\hline Target 2-feature mismatch, distractor match & $1188(47)$ & $229(24)$ & $318(53)$ \\
\hline Target 2-feature mismatch, distractor mismatch & $1199(64)$ & $456(65)$ & $405(91)$ \\
\hline
\end{tabular}

Second-pass reading time 


\begin{tabular}{rccc} 
Target match, distractor match & $873(97)$ & $223(30)$ & $265(36)$ \\
Target match, distractor mismatch & $799(93)$ & $224(27)$ & $253(36)$ \\
1-feature mismatch, distractor match & $881(98)$ & $318(34)$ & $266(38)$ \\
eature mismatch, distractor mismatch & $1044(106)$ & $339(34)$ & $234(26)$ \\
2-feature mismatch, distractor match & $925(99)$ & $212(25)$ & $287(36)$ \\
\hline
\end{tabular}

Table 9. Summary of statistical analyses by region and measure in Experiment 3. Significant coefficients $(|t|>2)$ are in bold.

\begin{tabular}{|c|c|c|c|c|c|c|c|c|c|}
\hline & \multirow{2}{*}{\multicolumn{3}{|c|}{ Pre-critical }} & \multicolumn{3}{|c|}{ Regions } & \multirow{2}{*}{\multicolumn{3}{|c|}{ Spillover }} \\
\hline & & & & \multicolumn{3}{|c|}{ Critical } & & & \\
\hline & $\widehat{\beta}$ & SE & $\mathrm{t}$ & $\widehat{\beta}$ & SE & $\mathrm{t}$ & $\widehat{\beta}$ & SE & $\mathrm{t}$ \\
\hline \multicolumn{10}{|l|}{ First pass reading time } \\
\hline$\overline{1 \text {-feature grammaticality }}$ & -0.01 & 0.06 & -0.21 & -0.09 & 0.23 & -0.40 & 0.29 & 0.27 & 1.08 \\
\hline 2-feature grammaticality & -0.02 & 0.06 & -0.30 & 0.27 & 0.23 & 1.15 & -0.26 & 0.27 & 1.08 \\
\hline Distractor match & 0.00 & 0.06 & 0.03 & -0.39 & 0.23 & -1.67 & 0.38 & 0.27 & 1.40 \\
\hline 1-feature grammaticality $\times$ distractor match & -0.11 & 0.09 & -1.17 & 0.45 & 0.33 & 1.37 & -0.51 & 0.38 & -1.33 \\
\hline 2 -feature grammaticality $\times$ distractor match & 0.03 & 0.09 & 0.35 & -0.57 & 0.33 & -1.74 & 0.13 & 0.38 & 0.34 \\
\hline 1 -feature attraction & -0.10 & 0.06 & -1.60 & 0.06 & 0.22 & 0.28 & -0.11 & 0.28 & -0.42 \\
\hline 2-feature attraction & 0.03 & 0.06 & 0.53 & -0.97 & 0.26 & -3.71 & 0.53 & 0.33 & 1.61 \\
\hline 1 -feature attraction $\times 2$-feature attraction & -0.14 & 0.09 & -1.57 & 1.03 & $\mathbf{0 . 3 3}$ & 3.12 & -0.65 & 0.39 & -1.60 \\
\hline \multicolumn{10}{|l|}{$\underline{\text { Right-bound reading time }}$} \\
\hline 1-feature grammaticality & -0.02 & 0.03 & -0.54 & -0.07 & 0.23 & -0.33 & 0.30 & 0.30 & 1.01 \\
\hline 2-feature grammaticality & -0.04 & 0.04 & -1.20 & 0.35 & 0.23 & 1.52 & -0.22 & 0.30 & -0.73 \\
\hline Distractor match & 0.01 & 0.03 & 0.39 & -0.39 & 0.23 & -1.70 & 0.40 & 0.31 & 1.28 \\
\hline 1-feature grammaticality $\times$ distractor match & -0.06 & 0.05 & -1.16 & 0.48 & 0.33 & 1.47 & -0.49 & 0.41 & -1.18 \\
\hline 2-feature grammaticality $\times$ distractor match & 0.04 & 0.05 & 0.80 & -0.66 & 0.33 & -1.98 & 0.10 & 0.47 & 0.22 \\
\hline 1 -feature attraction & -0.05 & 0.04 & -1.20 & 0.08 & 0.22 & 0.39 & -0.07 & 0.29 & -0.26 \\
\hline 2-feature attraction & 0.05 & 0.04 & 1.43 & -1.06 & 0.27 & -3.92 & 0.51 & 0.34 & 1.50 \\
\hline 1 -feature attraction $\times 2$-feature attraction & -0.10 & 0.05 & -1.90 & 1.15 & 0.33 & 3.45 & -0.59 & 0.40 & -1.48 \\
\hline \multicolumn{10}{|l|}{ Regression path duration } \\
\hline 1-feature grammaticality & -0.01 & 0.04 & -0.24 & -0.03 & 0.24 & -0.16 & 0.30 & 0.32 & 0.94 \\
\hline 2-feature grammaticality & -0.02 & 0.04 & -0.51 & 0.42 & 0.24 & 1.73 & -0.19 & 0.33 & -0.57 \\
\hline Distractor match & 0.03 & 0.04 & 0.66 & -0.41 & 0.24 & -1.73 & 0.41 & 0.33 & 1.23 \\
\hline 1-feature grammaticality $\times$ distractor match & -0.04 & 0.06 & -0.73 & 0.49 & 0.34 & 1.43 & -0.47 & 0.44 & -1.06 \\
\hline 2-feature grammaticality $\times$ distractor match & -0.00 & 0.06 & -0.05 & -0.70 & 0.34 & -2.04 & 0.09 & 0.51 & 0.19 \\
\hline 1 -feature attraction & -0.01 & 0.05 & -0.31 & -0.41 & 0.23 & -1.76 & -0.04 & 0.31 & -0.12 \\
\hline 2-feature attraction & 0.02 & 0.04 & 0.55 & -1.12 & 0.28 & -3.99 & 0.51 & 0.37 & 1.40 \\
\hline 1 -feature attraction $\times 2$-feature attraction & -0.04 & 0.06 & -0.64 & 1.19 & 0.34 & 3.45 & -0.56 & 0.43 & -1.37 \\
\hline \multicolumn{10}{|l|}{ Second-pass reading time } \\
\hline 1-feature grammaticality & 0.52 & 0.31 & 1.66 & 0.93 & 0.29 & 3.15 & 0.12 & 0.30 & 0.40 \\
\hline 2-feature grammaticality & 1.15 & 0.31 & 3.60 & 1.28 & 0.29 & 4.32 & 0.31 & 0.30 & 1.01 \\
\hline Distractor match & 0.20 & 0.31 & 0.64 & -0.19 & 0.29 & -0.64 & 0.35 & 0.30 & 1.16 \\
\hline 1-feature grammaticality $\times$ distractor match & -0.44 & 0.44 & -0.99 & 0.03 & 0.41 & 0.07 & -0.65 & 0.43 & -1.49 \\
\hline 2 -feature grammaticality $\times$ distractor match & -0.97 & 0.45 & -2.17 & -0.89 & 0.42 & -2.13 & -0.01 & 0.43 & -0.02 \\
\hline 1-feature attraction & -0.25 & 0.38 & -0.65 & -0.20 & 0.28 & -0.71 & -0.29 & 0.31 & -0.95 \\
\hline 2-feature attraction & -0.79 & 0.31 & -2.52 & -1.08 & 0.31 & -3.49 & 0.32 & 0.31 & 1.02 \\
\hline 1-feature attraction $\times 2$-feature attraction & 0.53 & 0.44 & 1.19 & 0.92 & 0.42 & 2.19 & -0.62 & 0.44 & -1.40 \\
\hline
\end{tabular}

Figure 4. Mean raw reading times by measure at the critical region in Experiment 3. Error bars indicate standard error of the mean. 

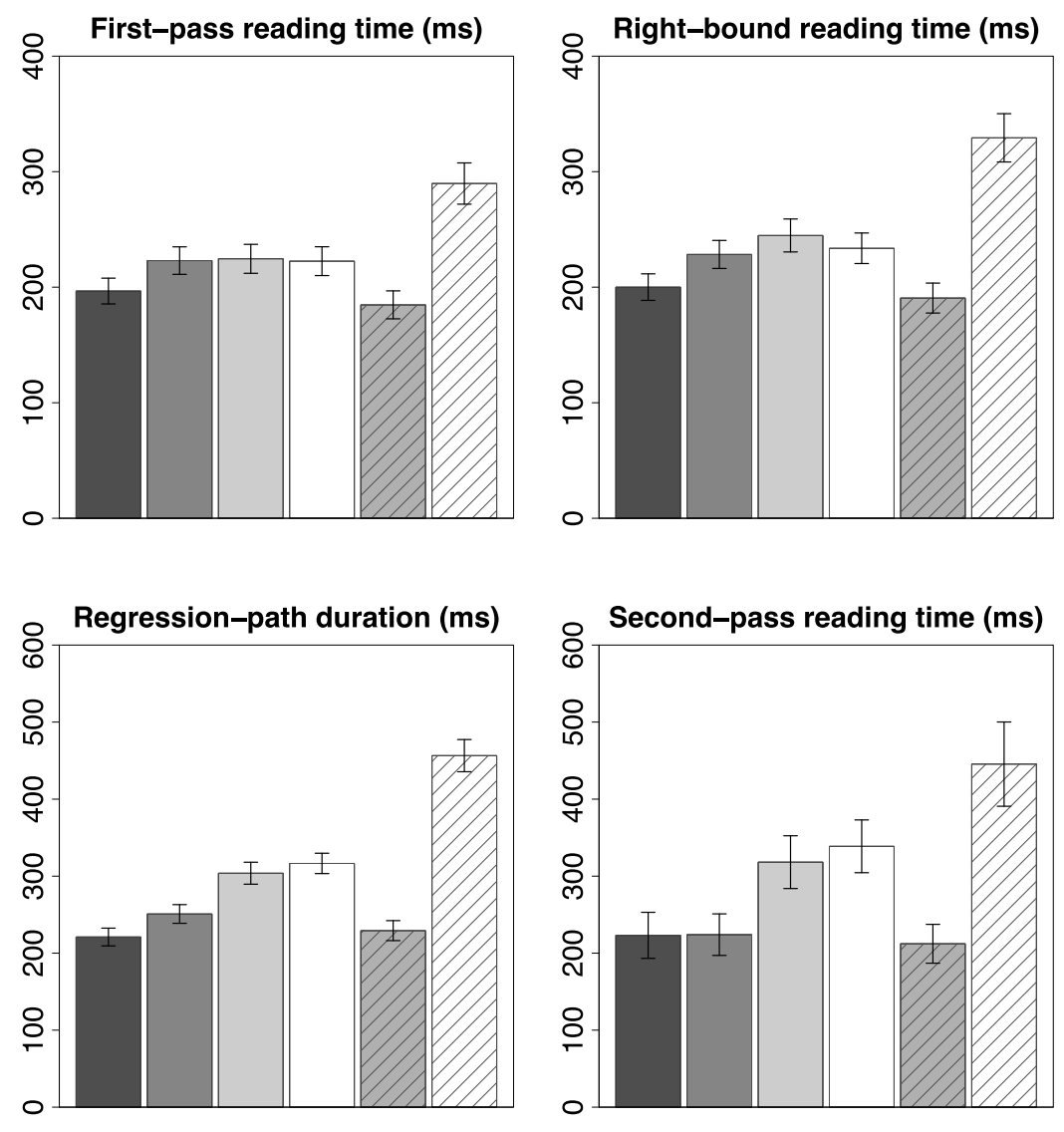

- Target match, distractor match

$\square$ Target match, distractor mismatch

1-feature mismatch, distractor match

1-feature mismatch, distractor mismatch

2-feature mismatch, distractor match

2-feature mismatch, distractor mismatch

\section{Discussion}

Experiment 3 was designed to test whether reflexive attraction is driven by the use of animacy features in retrieval or more generally by the strength of the reflexive-antecedent match. To achieve this, we held constant the animacy match between the potential licensors and reflexive, and deployed a feature combination involving gender and number. Results showed the same modulation of the attraction effect seen in Experiment 1: when the reflexive mismatched the target subject in just one feature, there was no attraction, but when it mismatched in two features, clear attraction effects were found. These findings demonstrate that the presence or absence of reflexive attraction does not rely on animacy features in retrieval, favoring an account based on 
the strength of the reflexive-antecedent match. We develop this proposal further in the General Discussion.

\section{General Discussion}

\section{Summary of findings}

In this paper, we tested the prediction that reflexives should be more susceptible to attraction when the reflexive and target subject mismatch in multiple features. This prediction was motivated by previous empirical contrasts (e.g., Parker et al., 2015) and computational simulations (reported in the Introduction). Across three eye-tracking experiments, we compared contexts where the target subject and a direct object reflexive mismatched in one feature, e.g., gender or number, and in two features, e.g., animacy + gender (Experiment 1), animacy + number (Experiment 2), and gender + number (Experiment 3). Results showed that reflexives are indeed susceptible to attraction, but only selectively. When the reflexive mismatched the true subject in just one feature, we consistently observed no attraction, but when it mismatched in more features, strong attraction effects were found. These results suggest the following generalization: when the target subject is an especially poor match to the retrieval cues at the reflexive, retrieval is sensitive to items that are grammatically irrelevant for the purpose of dependency formation.

\section{Survey of empirical contrasts}

Previous studies have shown that attraction effects are consistently found for agreement, but not for reflexives (see Dillon, 2014, for a review). We showed that reflexives are in fact susceptible to attraction by making a minimal change to the number of feature mismatches between the target subject and reflexive. To illustrate how our results diverge from previous studies, Figure 6 compares the findings from Experiments 1-3 of the current study to the effects reported in previous studies, e.g., Sturt (2003; labeled 'S'), Cunnings and Sturt (2014; labeled 'C\&S'), and Dillon et al. (2013; labeled 'D et al.'). ${ }^{3}$ The results from our 1-feature mismatch conditions are closely aligned with the results of previous studies that manipulated only one feature, showing a lack of attraction. By contrast, the results from our 2-feature mismatch conditions diverge

3 Figure 6 reports late eye-tracking measures, e.g., second-pass, total time, since these measures consistently show attraction for both agreement and reflexives. 
sharply from those studies, revealing attraction effects for reflexives that are as strong as those observed for agreement, if not stronger.

Figure 6. Comparison of the interference effects across Experiments 1-3 (labeled as P\&P) with the effects reported in Sturt (2003; labeled as 'S'), Cunnings \& Sturt (2014; labeled as C\&S), and Dillon et al. (2013; labeled as 'D et al.).

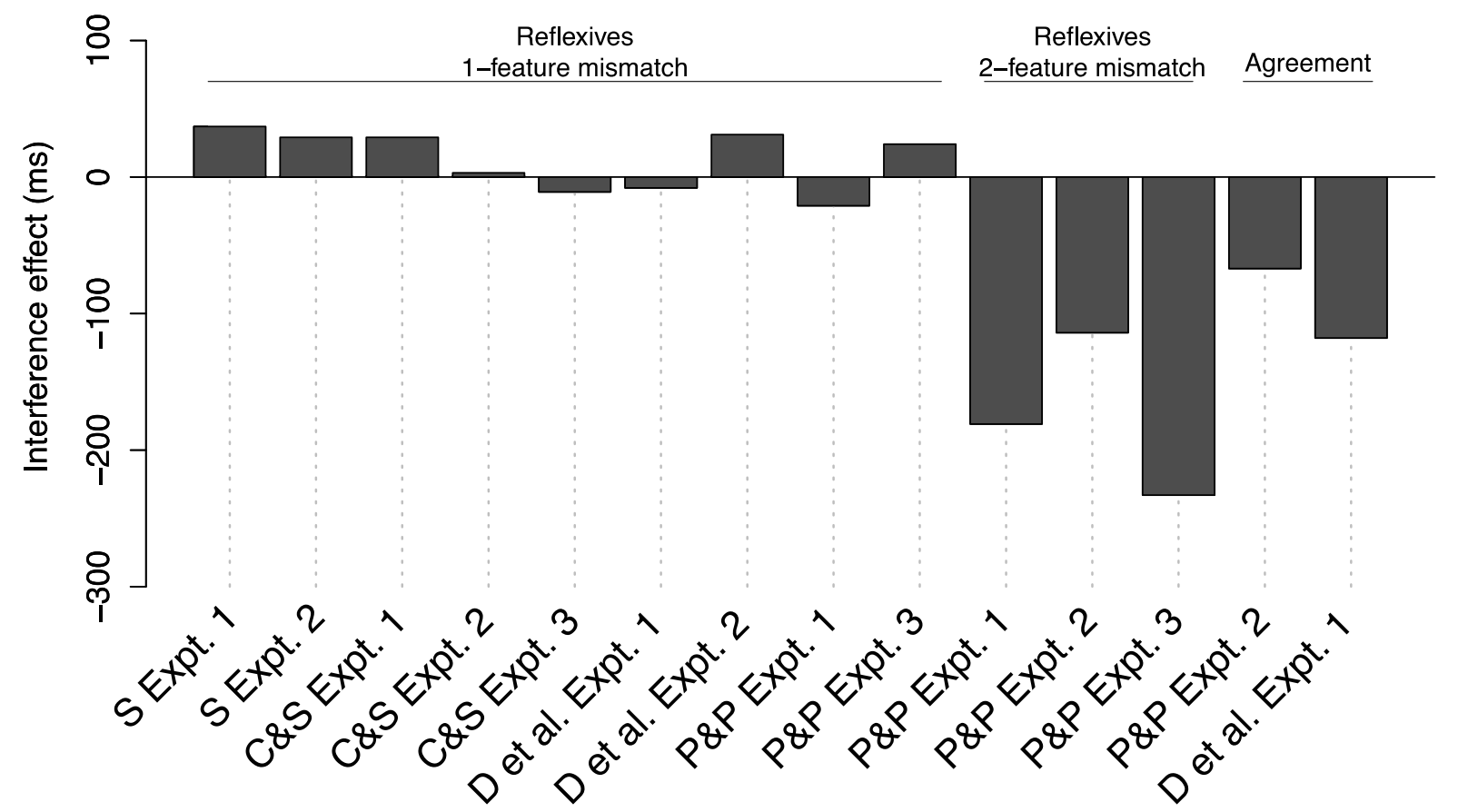

A few studies have reported interference in reflexive processing, but the effects reported in those studies are not representative of typical attraction effects found with agreement. Cunnings and Felser (2013) manipulated the gender match with direct object reflexives, and observed an attraction effect. However, this effect obtained only in early eye-tracking measures after a partitioning of the participants based on working memory span. King, Andrews, and Wagers (2012) also manipulated gender and showed that attraction obtains specifically for reflexives embedded in a post-verbal prepositional phrase (PP). However, reflexives in this position may be associated with different licensing conditions than those that govern direct object reflexives. Patil et al. (2016) manipulated the gender match with direct object reflexives, and observed a marginal effect of attraction in late eye-tracking measures. The effects reported in these studies are smaller and less consistent than the effects reported in the present study. For instance, Cunnings and 
Felser (2013) reported a facilitation effect of $19 \mathrm{~ms}$ in early eye-tracking measures, whereas in the present study, the facilitation effects in early measures ranged from $46 \mathrm{~ms}$ to $105 \mathrm{~ms}$.

The current results have several consequences for theories of memory access in sentence comprehension. Most importantly, our findings are consistent with the claim that there is a direct relation between grammatical constraints and their implementation as retrieval cues. Previous empirical contrasts between agreement and reflexive licensing (e.g., Dillon et al., 2013) motivated the claim that morphological cues play a different role in retrieval for the two dependencies, despite similar agreement constraints. However, the finding that reflexives and agreement are both susceptible to attraction suggests that they both recruit morphological agreement features as retrieval cues, as expected if there is a uniform mapping from grammatical constraints to retrieval cues. Of course, this does not explain why agreement attraction effects are routinely found in 1-feature mismatch contexts, whereas a stronger mismatch is required to reliably elicit reflexive attraction effects. We return to this issue below.

We argue that the current results are best captured in a cue-based parsing architecture where agreement and reflexives deploy the same memory access mechanism. Specifically, we suggest that agreement and reflexives both engage a cue-based retrieval mechanism that uses a combination of structural and non-structural (e.g., morphological) cues to access a licensor in content-addressable memory. This account is consistent with the general assumption that all linguistic dependencies are uniformly resolved using an error-prone, cue-based retrieval mechanism, as suggested in previous research (e.g., McElree, 2000; McElree et al., 2003; Lewis \& Vasishth, 2005).

\section{Why do subject-verb agreement and reflexive-antecedent dependencies differ?}

We suggested that agreement and reflexives deploy the same memory access mechanism and that there is a uniform mapping from grammatical constraints to retrieval cues. On this view, both agreement and reflexives should show identical attraction profiles. However, they are differentially susceptible to attraction: environments that exhibit strong agreement attraction effects, e.g., 1-feature mismatch contexts, do not exhibit reflexive attraction.

The varying strength of distractor effects in subject-verb and reflexive-antecedent dependencies could reflect baseline activation differences of the subject noun for the two dependencies. It is assumed in cue-based models (e.g., ACT-R; Lewis \& Vasishth, 2005) that 
retrieval targets heads of arguments, rather than entire arguments. In the sentences that we tested, the subject head noun is retrieved at the main verb for subject-verb integration immediately before the critical retrieval at the reflexive. This gives the subject head noun an activation boost that could reduce interference from the distractor during retrieval for reflexive licensing. No such difference is expected for subject-verb agreement, since the critical retrieval for agreement licensing occurs before subject-verb integration (see Dillon et al., 2013, for discussion).

We argue that fluctuations of activations cannot capture the observed contrasts. First, previous work using computational simulations has shown that baseline activation differences are insufficient to account for the lack of attraction with reflexives (Dillon et al., 2013). Second, the assumption that only the head of the subject NP is reactivated at the verb, rather than the entire argument, is neither obvious nor well-motivated. It is more likely that retrieval for subjectverb integration targets the entire argument, which would reactivate both the head and distractor contained inside.

We suggest instead that the contrast between subject-verb agreement and reflexiveantecedent dependencies with respect to attraction reflects differences in cue weightings for the two dependencies, based on the predictability of the dependency. Specifically, we suggest that the selectivity of reflexive attraction may reflect a weighted cue-combinatorics scheme in which structural cues are weighted more strongly than morphological cues. Weighting structural cues over morphological cues maximizes the probability of retrieving the structurally accessible target and minimizes attraction from structurally irrelevant items, even when the target is not a perfect match, e.g., 1-feature mismatch. However, the finding that reflexives are susceptible to attraction with stronger reflexive-antecedent mismatches, e.g., 2-feature mismatch, implies that the weighting for structural cues is not so strong that they serve a gating function (cf. Van Dyke \& McElree, 2011), so that only the target is considered.

To make this account explicit, we simulated cue weighting for reflexive licensing. Using the same computational model described in the Introduction, we systematically manipulated the weight assigned to structural cues relative to non-structural cues. Specifically, we implemented an agreement-based retrieval model for reflexive processing which used a mixture of syntactic and agreement features as cues to retrieve the subject. The simulations included weighted structural cues for local subject, which targeted the subject position of the current clause, and agreement cues for person, number, gender, and animacy (following Dillon et al., 2013). 
Figure 5 provides a plot of the predicted retrieval error rates as a function of structural cue weighting. When structural cues are weighted equally to non-structural cues, widespread attraction effects are predicted for both the 1- and 2-feature mismatch conditions. By contrast, when the weighting for structural cues is at its strongest, no attraction effects are predicted for either the 1- or 2-feature mismatch conditions. Interestingly, we find that at intermediate values, the model accurately predicts the absence of an attraction effect in the 1-feature mismatch condition and the presence of an attraction effect in the 2-feature mismatch condition. These results demonstrate that it is possible to capture both the presence and absence of reflexive attraction from the same retrieval mechanism by preferentially weighting structural cues over morphological cues.

Figure 5. Predicted retrieval error rates for 1-feature (solid) and 2-feature (dashed) mismatch conditions as a function of structural cue weighting.

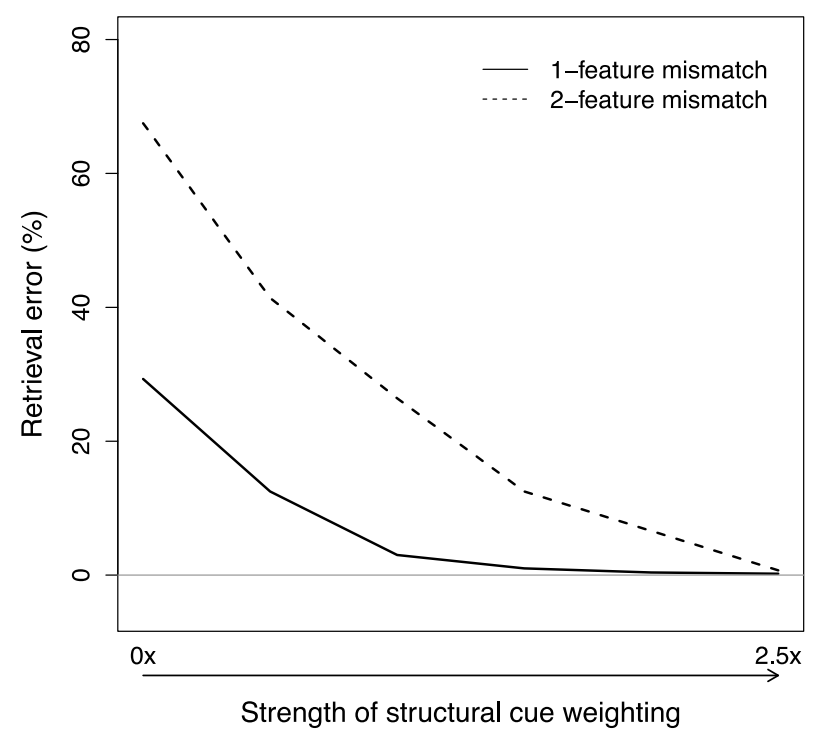

The modeling results raise two concerns about the nature of retrieval for dependency formation. First, we found that it is possible to model the distribution of attraction effects for reflexives using the same retrieval architecture and the same retrieval cues, but by varying the weighting of structural cues. However, we have not offered any way of guaranteeing that comprehenders should choose the specific weightings that capture the observed experimental contrasts. Further research is necessary to determine how learners converge on the correct 
weighting scheme. Second, it remains unclear how the parser determines when it should prioritize syntactic cues over morphological cues. The finding that agreement shows strong attraction effects, even in a 1-feature mismatch context, implies that structural cues are not weighted more strongly for agreement.

We suggest that the priority for structural cues is determined by the predictability of the dependency. Specifically, we argue that structural cues are prioritized when retrieval is triggered as part of normal resolution for unpredictable dependencies, such as those involving reflexives, but they are not prioritized when retrieval is triggered by a prediction error, such as in the case of subject-verb agreement. Previous work has argued that retrieval for subject-verb agreement functions as a repair mechanism triggered by the detection of a number prediction error (e.g., Lago et al., 2015; Wagers et al., 2009). On this view, the subject NP predicts the number of the verb, but when the verb form violates that prediction, the parser engages a cue-based retrieval process to recover a number matching noun to license agreement. The prediction error could lead the parser to be uncertain about the accuracy of the existing structural encoding and, hence, less reliant on syntactic cues during retrieval. Relying less on syntactic cues should increase the opportunity for attraction errors from number matching items in locations that are syntactically irrelevant for dependency formation. Reflexive anaphors, by contrast, cannot be anticipated as reliably as subject-verb agreement, and are less likely to violate expectations in a way that would impact priority for structural cues. Retrieval is required as part of normal resolution, and syntactic cues should remain prioritized over morphological cues. On this view, structural cues are weighted more strongly than morphological cues by default as part of normal resolution for dependencies that define their licensors in structural terms. This includes both reflexive licensing and subject-verb agreement. But the priority for structural cues can be neutralized when retrieval is triggered by a prediction error that impacts the parser's confidence about the accuracy of the existing structural encoding.

Our proposal is supported by the timing profiles observed in Experiments 2 and 3, which show that detection of grammaticality violations precedes attraction effects for agreement, and that attraction effects are simultaneous with the grammaticality effects for reflexives. For agreement, we observed a main effect of grammaticality in early measures, e.g., first-pass reading times, followed by attraction in later measures. This timing profile is predicted under accounts that posit that retrieval is triggered by the initial detection of a violated agreement 
prediction, and replicates previous studies showing that grammaticality effects show an earlier onset than attraction effects (e.g., Lago et al., 2015; Tucker et al., 2016). Under this account, the grammaticality effect in early measures reflects detection of an agreement prediction error, triggering the error-driven retrieval processes that gave rise to the attraction effects observed in later measures. Reflexives, by contrast generally showed attraction from the earliest stages of processing, e.g., first-pass reading times, since they must engage retrieval from the start as part of normal resolution. The exception to this pattern was Experiment 1, where all effects for reflexives were observed in later measures.

The current proposal raises the question of whether similar effects of cue-weighting would be observed in languages other than English. Would we see such effects in languages with a rich morphology system? Lago et al. (2015) conducted a distributional analysis of agreement attraction effects in Spanish, a language which has richer agreement morphology than English, and showed that these effects are similar in their magnitude and distribution to those previously observed in English. Based on these findings, we find no reason to assume that our proposal should be implemented differently in other languages. ${ }^{4}$ But, we leave this issue to future research.

The different timing profiles for attraction reported in the current study, taken together with previous empirical contrasts (e.g., Parker et al., 2015), provide two insights into the nature of retrieval for dependency formation. First, they suggest that retrieval prioritizes structural cues over non-structural cues, and that susceptibility to attraction might be based on the predictability of the dependency. Second, the current results provide further evidence for the proposal that retrieval for agreement licensing functions as a repair mechanism triggered by the detection of an agreement prediction error (Lago, et al., 2015; Wagers et al., 2009). The finding that grammaticality violations precede attraction effects for agreement is unexpected under accounts that retrieval is always engaged at the verb, and also under percolation accounts (e.g., Eberhard,

\footnotetext{
${ }^{4}$ Martin and colleagues tested anaphoric dependencies involving ellipsis in Spanish, and reported that the gender of a grammatically irrelevant attractor modulated ERP responses associated with ellipsis resolution, even in grammatical sentences (Martin, Nieuwland, \& Carrieras, 2012, 2013). These findings do not challenge our proposal for several reasons. First, ellipsis is less structurally constrained than the reflexive-antecedent dependencies that we tested. In fact, many formal theories assume that ellipsis is semantically licensed, which could impact real-time processing (see Phillips \& Parker, 2014, for a review). Second, the effects differ in important ways from previous demonstrations of attraction. Martin and colleagues showed a sustained negativity and increased P600, rather than the reduced P600 shown in previous tests (e.g., Tanner et al., 2012, 2014; see also, Xiang et al., 2009).
} 
Cutting, \& Bock, 2005; Franck, Vigliocco, \& Nicol, 2002; Pearlmutter et al., 1999; see Introduction for discussion). These accounts posit that agreement can be computed only after retrieval has taken place, and incorrectly predict simultaneous effects of grammaticality violations and attraction.

\section{Conclusion}

In this paper, we tested the prediction that reflexive attraction reflects the strength of the reflexive-antecedent feature match. Across three eye-tracking experiments, we showed that it is possible to systematically control where attraction effects do and do not occur for reflexive licensing. Furthermore, we showed based on computational simulations that it is possible to derive both the presence and absence of reflexive attraction from the same retrieval mechanism using a cue-combinatorics scheme that preferentially weights structural cues over morphological cues. Finally, we proposed an account of why agreement and reflexives differ with respect to attraction, based on the predictability of the dependency. Ultimately, these results provide further support for the claim that there is a direct relation between grammatical constraints and their implementation as retrieval cues (see Phillips et al., 2011, and Dillon, et al., 2013, for discussion) and that all linguistic dependencies are uniformly resolved using an error-prone, cue-based retrieval mechanism (e.g., McElree, 2000; McElree et al., 2003; Lewis \& Vasishth, 2005).

\section{Acknowledgments}

We would like to thank Brian Dillon, Norbert Hornstein, Dave Kush, and Sol Lago for helpful discussions. This work was supported in part by NSF-IGERT grant DGE-0801465 to the University of Maryland and by NSF grant BCS-0848554 to Colin Phillips.

\section{References}

Anderson, J. R., \& Reder, L. M. (1999). The fan effect: New results and new theories. Journal of Experimental Psychology: General, 128, 186-197.

Anderson, J. R., (1990). The Adaptive Character of Thought. Erlbaum, Hillsdale, NJ.

Anderson, J. R. (1974). Retrieval of propositional information from long-term memory. Cognitive Psychology, 6, 451-474. 
Baayen, R. H., Davidson, D., \& Bates, D. (2008). Mixed-effects modeling with crossed random effects for subjects and items. Journal of Memory and Language, 59, 390-412.

Barr, D. J., Levy, R., Scheepers, C., \& Tily, H. J. (2013). Random effects structure for testing interactions in linear mixed-effects models. Frontiers in Psychology, 4, 328.

Bates, D., Maechler, M., \& Bolker, B. (2014). lme4: Linear mixed-effects models using S4 classes. Retrieved from http://CRAN.R-project.org/package=lme4

Box, G. E. P. \& Cox, D. R. (1964). An analysis of transformations. Journal of the Royal Statistical Society. Series B (Methodological), 26, 211-252.

Clifton, C., Frazier, L., \& Deevy, P. (1999). Feature manipulation in sentence comprehension. Rivista di Linguistica, 11, 11-39.

Cunnings, I \& Sturt, P. (2014). Coargumenthood and the processing of reflexives. Journal of Memory and Language, 75, 117-139.

Cunnings, I., \& Felser, C. (2013). The role of working memory in the processing of reflexives. Language and Cognitive Processes, 28, 188-219.

Dillon, B. (2014). Syntactic Memory in the Comprehension of Reflexive Dependencies: an Overview. Language and Linguistics Compass, 5, 171-187.

Dillon, B., Mishler, A., Sloggett, S., \& Phillips, C. (2013). Contrasting intrusion profiles for agreement and anaphora: Experimental and modeling evidence. Journal of Memory and Lanuage, 69, 85-103.

Dillon, B. (2011). Structured access in sentence comprehension. Ph.D. Dissertation, University of Maryland.

Drenhaus, H., Saddy, D., \& Frisch, S. (2005). Processing negative polarity items: When negation comes through the backdoor. In S. Kepser \& M. Reis (Eds.), Linguistic evidence: Empirical, theoretical, and computational perspectives (pp. 145-165). Berlin: de Gruyter.

Gelman, A., \& Hill, J. (2007). Data analysis using regression and multilevel/hierarchical models. Cambridge: Cambridge University Press.

King, J., Andrews, C., \& Wagers, M. W. (2012). Do reflexives always find a good antecedent for themselves? Poster presented at the $25^{\text {th }}$ annual CUNY Conference on Human Sentence Processing. New York, NY.

Kush, D. \& Phillips, C. (2014). Local anaphor licensing in an SOV langauge: implications for retrieval strategies. Frontiers in Psychology, 5, 1-12. 
Kreiner, H., Sturt, P., \& Garrod, S. (2008). Processing definitional and stereotypical gender in reference resolution: Evidence from eye-movements. Journal of Memory and Language, 58, 239-261.

Lago, S., Shalom, D., Sigman, M., Lau, E., \& Phillips, C. (2015). Agreement processes in Spanish comprehension. Journal of Memory and Language, 82, 133-149.

Lewis, R. L., \& Vasishth, S. (2005). An activation-based model of sentence processing as skilled memory retrieval. Cognitive Science, 29, 375-419.

Lewis, R. L., Vasishth, S., \& Van Dyke, J. A. (2006). Computational Principles of Working Memory in Sentence Comprehension. Trends in Cognitive Science, 10, 447-454.

Martin, A. E. \& McElree, B. (2008). A content-addressable pointer mechanism underlies comprehension of verb-phrase ellipsis. Journal of Memory and Language, 58, 879-906.

McElree, B. (2000). Sentence comprehension is mediated by content-addressable memory structures. Journal of Psycholinguistic Research, 29, 155-200.

McElree, B. (2006). Accessing recent events. In B. H. Ross (Ed). The psychology of learning and motivation (Vol. 46, pp. 155-200). San Diego: Academic Press.

McElree, B., Foraker, S., \& Dyer, L. (2003). Memory structures that subserve sentence comprehension. Journal of Memory and Language, 48, 67-91.

Nairne, J. S., Van Arsdall, J. E., Pandeirada, J. N. S., Cogdill, M., \& LeBreton, J.M. (2013). Adaptive memory: the mnemonic value of animacy. Psychological Science, 24, 2099-2105.

Nairne, J. S. (1988). A framework for interpreting recency effects in immediate serial recall. Memory \& Cognition, 16, 343-352.

Nairne, J. S. (1990). A feature model of immediate memory. Memory \& Cognition, 18, 251-269.

Nicol, J., \& Swinney, D. (1989). The role of structure in coreference assignment during sentence comprehension. Journal of Psycholinguistic Research, 18, 5-19.

Patil, U., Vasishth, S., \& Lewis, R. L. (2016). Retrieval Interference in Syntactic Processing: The Case of Reflexive Binding in English. Frontiers in Psychology, 7, 1-18.

Parker, D. \& Phillips, C. (2016). Negative polarity illusions and the format of hierarchical encodings in memory. Cognition, 157, 321-339.

Parker, D., Lago, S., \& Phillips, C. (2015). Interference in the processing of adjunct control. Frontiers in Psychology, 6, 1-13. 
Pearlmutter, N., Garnsey, S., \& Bock, K. (1999). Agreement processes in sentence comprehension. Journal of Memory and Language, 41, 427-456.

Patson, N. D. \& Husband, M. E. (2015). Misinterpretations in agreement and agreement attraction. The Quarterly Journal of Experimental Psychology, 69, 950-971.

Phillips, C. \& Parker, D. (2014). The psycholinguistics of ellipsis. Lingua, 151, 78-95.

Phillips, C., Wagers, M. W., \& Lau, E. F. (2011). Grammatical illusions and selective fallibility in real-time language comprehension. In J. Runner (Ed.), Experiments at the Interfaces (Vol. 37, pp. 147-180). Bingley, UK: Emerald Publications.

Pollard, C. \& Sag., I. (1992). Anaphors in English and the scope of binding theory. Linguistic Inquiry, 23, 261-303.

R Development Core Team. (2014). R: A language and environment for statistical computing. Vienna, Austria: R Foundation for Statistical Computing. Retrieved from http://www.Rproject.org.

Reinhart, T. \& Reuland, E. (1993). Reflexivity. Linguistic Inquiry, 24, 657-720.

Staub, A. (2010). Response time distributional evidence for distinct varieties of number attraction. Cognition, 114, 447-454.

Sturt, P. (2003). The time-course of the application of binding constraints in reference resolution. Journal of Memory and Language, 48, 542-562.

Tanner, D., Nicol, J., \& Brehm, L. (2014). The time-course of feature interference in agreement comprehension: multiple mechanisms and asymmetrical attraction. Journal of Memory and Language, 76, 195-215.

Tucker, M., Idrissi, A., \& Almeida, D. (2015). Attraction errors for gender in Modern Standard Arabic reading. Poster presented at the Architectures and Mechanisms for Language Processing (AMLaP) 2015 Conference. University of Valletta, Malta.

Van Arsdall, J. E., Nairne, J. S., Pandeirada, J. N. S., \& Blunt, J. R. (2013). Adaptive memory: animacy processing produces mnemonic advantages. Experimental Psychology, 60, 172-178.

Van Dyke, J. A. \& Lewis, R. L. (2007). Interference effects from grammatically unavailable constituents during sentence processing. Journal of Experimental Psychology: Learning, Memory, and Cognition, 33, 407-430.

Van Dyke, J. A., \& McElree, B. (2006). Retrieval interference in sentence comprehension. Journal of Memory and Language, 55, 157-166. 
Van Dyke, J. A., \& McElree, B. (2011). Cue-dependent interference in comprehension. Journal of Memory and Language, 65, 247-263.

Vasishth, S., Brüssow, S., Lewis, R. L., \& Drenhaus, H. (2008). Processing polarity: How the ungrammatical intrudes on the grammatical. Cognitive Science, 32, 685-712.

Wagers, M. W., Lau, E. F., \& Phillips, C. (2009). Agreement attraction in comprehension: Representations and processes. Journal of Memory and Language, 61, 206-237.

Xiang, M., Dillon, B., \& Phillips, C. (2009). Illusory licensing effects across dependency types: ERP evidence. Brain \& Language, 108, 40-55. 